\title{
Manual de Laboratorio de Electromagnetismo
}

ISBN (e) 978-958-8166-90-2

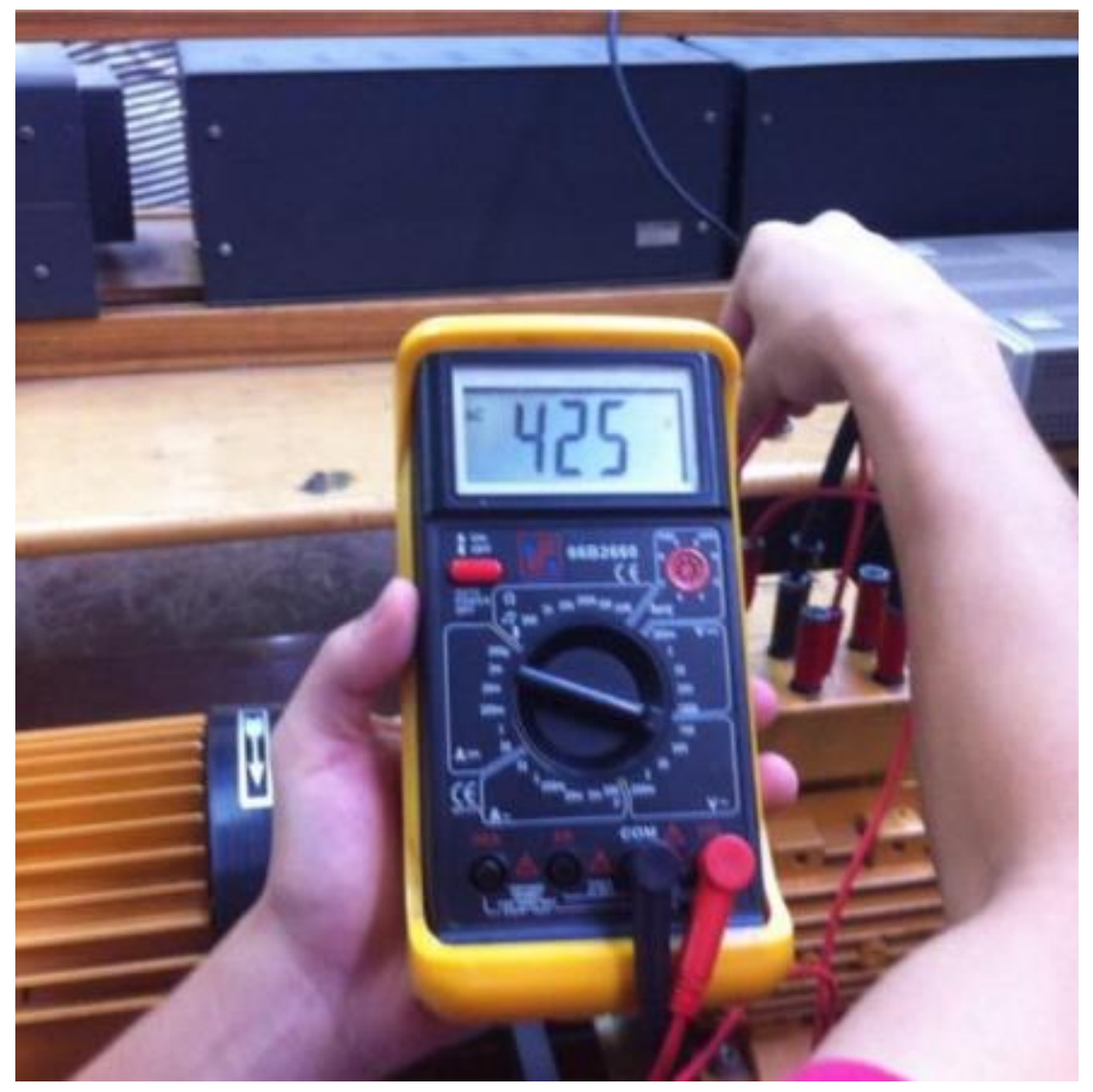

Ligia Beleño Montagut

Martha Lucía Barrera Pérez

Luis Alejandro Prada Martínez

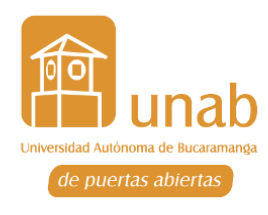




\title{
UNIVERSIDAD AUTÓNOMA DE BUCARAMANGA
}

Manual de Laboratorio de Electromagnetismo

ISBN (e) 978-958-8166-90-2

\author{
ALBERTO MONTOYA PUYANA \\ Rector \\ EULALIA GARCÍA BELTRÁN \\ Vicerrectora Académica \\ GILBERTO RAMÍREZ VALBUENA \\ Vicerrector administrativo y financiero \\ NÓHORA ISABEL NÁJERA ESTEBAN \\ Directora \\ Departamento de Matemáticas y Ciencias Naturales
}

LIGIA BELEÑO MONTAGUT

Departamento de Matemáticas y Ciencias Naturales

MARTHA LUCÍA BARRERA PÉREZ

Departamento de Matemáticas y Ciencias Naturales

LUIS ALEJANDRO PRADA MARTÍNEZ

Autores

JOSE OSCAR MACHADO ROMERO

Corrección de Estilo.

PUBLICACIONES UNAB

Producción

Universidad Autónoma de Bucaramanga

Avenida $42 \mathrm{~N}^{\circ} 48-11$

Bucaramanga, Colombia

www.unab.edu.co

Las opiniones contenidas en esta obra no vinculan a la Institución, sino que son exclusiva responsabilidad de los autores, dentro de los principios democráticos de la cátedra libre y la libertad de expresión consagrados en el artículo $3^{\circ}$ del Estatuto General de la Corporación Universidad Autónoma de Bucaramanga. 
TABLA DE CONTENIDO

Tabla de Contenido

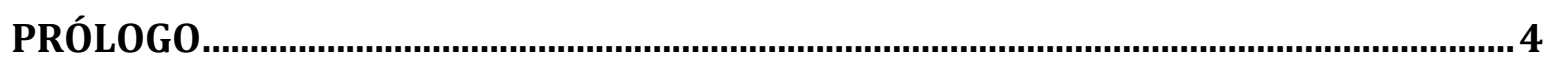

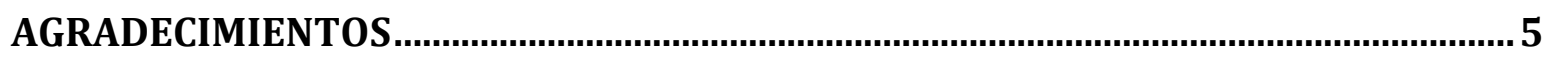

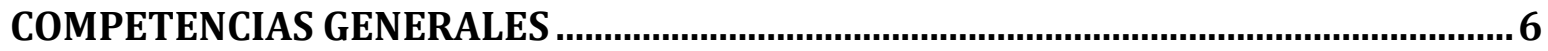

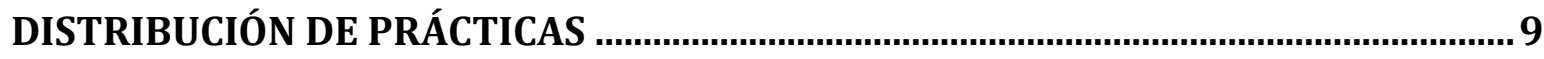

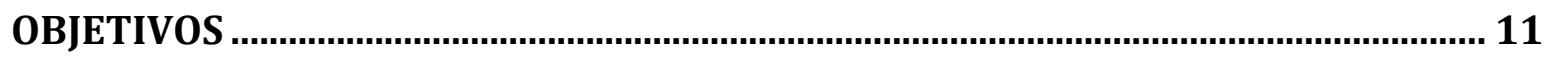

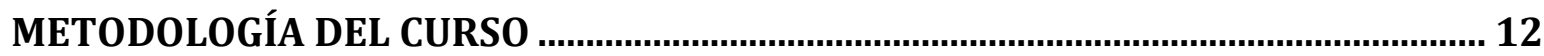

SESIÓN 1- LEY DE COULOMB................................................................................... 14

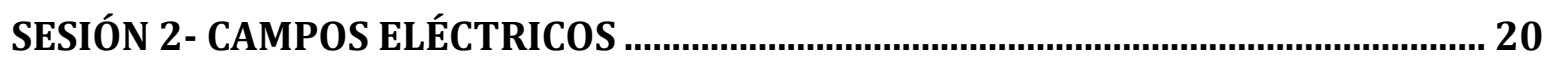

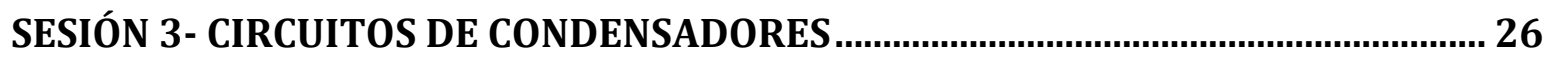

SESIÓN 4- RESISTIVIDAD ELÉCTRICA....................................................................... 34

SESIÓN 5- CIRCUITOS DE CORRIENTE CONTINUA.................................................... 41

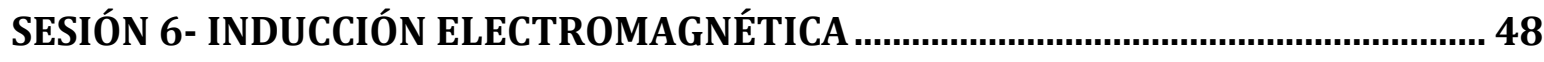

SESIÓN 7- CIRCUITOS SERIE DE CORRIENTE ALTERNA - RLC .................................. 53 


\section{Prólogo}

Este Manual de laboratorio es producto del análisis y trabajo continuo de los docentes de tiempo completo en la línea de física del departamento de Matemáticas y Ciencias Naturales. La finalidad de este manual es apoyar tanto a estudiantes como a docentes en el desarrollo del curso de Laboratorio de Electromagnetismo, de manera que reconozcan el entorno de trabajo y puedan preparar previamente la fundamentación requerida en cada una de las sesiones programadas.

El curso de laboratorio de Electromagnetismo complementa el conocimiento adquirido en el curso teórico de Electromagnetismo. Se espera que este libro sirva como guía para la realización de diversos experimentos que comprueben los principios fundamentales de la Electricidad y el Magnetismo.

Cada experimento se realizará en forma simultánea en ocho montajes que estarán dispuestos en cada mesa de trabajo para un número máximo de tres estudiantes por grupo. El laboratorio de Física dispone de todos los elementos requeridos para la realización de las prácticas descritas en este manual, en los que se incluyen fuentes de corriente continua y alterna, medidores de voltaje, corriente, resistencia, capacitancia, etc., galvanómetros, osciloscopios, transformadores, bobinas, entre otros.

Se espera que el estudiante prepare con anticipación la fundamentación teórica que se sugiere en cada guía con el fin de identificar más claramente los conceptos involucrados en la práctica correspondiente. Los cálculos, evaluación de errores y conclusiones serán el resultado del análisis de tablas y gráficas realizadas durante el experimento.

Esperamos que el uso de este material les permita hacer aportes con miras a mejorar lo propuesto. Sus ideas y sugerencias serán bien recibidas.

\section{Los autores}




\section{Agradecimientos}

Los autores agradecen a la Universidad Autónoma de Bucaramanga por el espacio académico de laboratorio el cual fortalece las habilidades de nuestros alumnos de ingeniería y a su vez representa un gran apoyo a los docentes en sus labores académicas.

Por otra parte se agradece a los compañeros y a los alumnos que con sus ideas y observaciones han participado de manera activa en este constante proceso de construcción.

De manera especial se valora el apoyo del Ingeniero Raúl Ortiz Rojas por su constante apoyo como auxiliar del laboratorio. 


\section{Competencias generales}

\section{Respecto al trabajo en equipo}

- $\quad$ Propender por el logro de los objetivos del equipo de trabajo, con el fin de cumplir con los compromisos que requiere el desarrollo de la práctica experimental.

- Valorar las ideas y opiniones de los demás compañeros del equipo, para construir el análisis del experimento a partir de las mismas.

- Colaborar activamente con sus compañeros del equipo con la propuesta de soluciones.

- Facilitar la comunicación, manteniendo relaciones productivas y respetuosas con los demás compañeros.

\section{Respecto a la iniciativa}

- Participar activamente, aportando ideas y estimulando a sus compañeros de grupo a trabajar de forma conjunta y proactiva.

- Tomar decisiones inmediatas cuando se detecte algún error en el procedimiento realizado, con el fin de reducir el riesgo de obtener resultados inconsistentes en el análisis del experimento. - $\quad$ Analizar con profundidad las situaciones propuestas en las guías de laboratorio.

\section{Respecto a la flexibilidad}

- $\quad$ Adaptarse a cada situación que se presente durante el desarrollo de la experiencia de laboratorio.

- $\quad$ Propender por la tolerancia hacia los diversos puntos de vista de sus compañeros, con el fin de mantener la armonía y favorecer la toma de las mejores decisiones del grupo y así alcanzar las metas propuestas. 


\section{Respecto a la comunicación}

- Facilitar la comunicación con los demás miembros del grupo, socializando los resultados obtenidos durante la práctica.

- Escuchar activamente a sus compañeros, aportando la retroalimentación necesaria y analizando las participaciones de los demás, para el logro de los objetivos de la experiencia realizada.

\section{Respecto a la responsabilidad}

- $\quad$ Comprometerse con todas las actividades previas a la realización del experimento, así como durante el desarrollo del mismo.

- Gestionar el tiempo del desarrollo de la práctica con el fin de poder entregar los resultados del informe dentro del plazo definido en el reglamento del laboratorio de física.

- $\quad$ Asumir con responsabilidad los errores cometidos por su equipo de trabajo, ya sea en la toma de mediciones, en el manejo de equipos y en la realización del informe, estableciendo con prontitud los correctivos necesarios para enmendarlos.

\section{Respecto al conocimiento}

- Expresar e interpretar conceptos y opiniones de forma oral y escrita, de acuerdo al contexto, con un buen manejo del vocabulario y aplicando correctamente las normas gramaticales del lenguaje. Análisis de un modelo específico al cual dará explicación con base en la toma de datos y análisis gráfico y de resultados. 
- Desarrollar y aplicar el razonamiento matemático para la formulación de modelos que permitan explicar los fenómenos naturales estudiados en cada una de las prácticas de laboratorio. - Utilizar las herramientas tecnológicas para el tratamiento y el uso de la información obtenida en la toma de mediciones, con el fin de producir y presentar el análisis de los resultados en el reporte final de cada laboratorio. 
Distribución de prácticas

\begin{tabular}{|c|c|c|c|c|c|c|c|c|}
\hline $\begin{array}{l}\text { Sesió } \\
\rightarrow \\
\downarrow_{\text {gru }} \\
\text { po }\end{array}$ & 1 & 2 & 3 & 4 & 5 & 6 & 7 & 8 \\
\hline I & $\begin{array}{l}\text { Ley de } \\
\text { Coulo } \\
\mathrm{mb}\end{array}$ & $\begin{array}{l}\text { Campo } \\
\mathrm{s} \\
\text { eléctric } \\
\text { os }\end{array}$ & $\begin{array}{l}\text { Circuitos } \\
\text { de } \\
\text { condensad } \\
\text { ores }\end{array}$ & $\begin{array}{l}\text { Resisti } \\
\text { vidad } \\
\text { eléctric } \\
\text { a }\end{array}$ & $\begin{array}{l}\text { Circuit } \\
\text { os de } \\
\text { c.c }\end{array}$ & $\begin{array}{l}\text { Inducc } \\
\text { ión } \\
\text { electro } \\
\text { magné } \\
\text { tica }\end{array}$ & $\begin{array}{l}\text { Circu } \\
\text { itos } \\
\text { RLC }\end{array}$ & $\begin{array}{l}\text { Exam } \\
\text { en } \\
\text { final } \\
*\end{array}$ \\
\hline II & $\begin{array}{l}\text { Ley de } \\
\text { Coulo } \\
\mathrm{mb}\end{array}$ & $\begin{array}{l}\text { Campo } \\
\mathrm{s} \\
\text { eléctric } \\
\text { os }\end{array}$ & $\begin{array}{l}\text { Circuitos } \\
\text { de } \\
\text { condensad } \\
\text { ores }\end{array}$ & $\begin{array}{l}\text { Resisti } \\
\text { vidad } \\
\text { eléctric } \\
\text { a }\end{array}$ & $\begin{array}{l}\text { Circuit } \\
\text { os de } \\
\text { c.c }\end{array}$ & $\begin{array}{l}\text { Inducc } \\
\text { ión } \\
\text { electro } \\
\text { magné } \\
\text { tica }\end{array}$ & $\begin{array}{l}\text { Circu } \\
\text { itos } \\
\text { RLC }\end{array}$ & $\begin{array}{l}\text { Exam } \\
\text { en } \\
\text { final } \\
*\end{array}$ \\
\hline III & $\begin{array}{l}\text { Ley de } \\
\text { Coulo } \\
\mathrm{mb}\end{array}$ & $\begin{array}{l}\text { Campo } \\
\mathrm{s} \\
\text { eléctric } \\
\text { os }\end{array}$ & $\begin{array}{l}\text { Circuitos } \\
\text { de } \\
\text { condensad } \\
\text { ores }\end{array}$ & $\begin{array}{l}\text { Resisti } \\
\text { vidad } \\
\text { eléctric } \\
\text { a }\end{array}$ & $\begin{array}{l}\text { Circuit } \\
\text { os de } \\
\text { c.c }\end{array}$ & $\begin{array}{l}\text { Inducc } \\
\text { ión } \\
\text { electro } \\
\text { magné } \\
\text { tica }\end{array}$ & $\begin{array}{l}\text { Circu } \\
\text { itos } \\
\text { RLC }\end{array}$ & $\begin{array}{l}\text { Exam } \\
\text { en } \\
\text { final } \\
*\end{array}$ \\
\hline IV & $\begin{array}{l}\text { Ley de } \\
\text { Coulo } \\
\mathrm{mb}\end{array}$ & $\begin{array}{l}\text { Campo } \\
\mathrm{s} \\
\text { eléctric } \\
\text { os }\end{array}$ & $\begin{array}{l}\text { Circuitos } \\
\text { de } \\
\text { condensad } \\
\text { ores }\end{array}$ & $\begin{array}{l}\text { Resisti } \\
\text { vidad } \\
\text { eléctric } \\
\text { a }\end{array}$ & $\begin{array}{l}\text { Circuit } \\
\text { os de } \\
\text { c.c }\end{array}$ & $\begin{array}{l}\text { Inducc } \\
\text { ión } \\
\text { electro } \\
\text { magné } \\
\text { tica }\end{array}$ & $\begin{array}{l}\text { Circu } \\
\text { itos } \\
\text { RLC }\end{array}$ & $\begin{array}{l}\text { Exam } \\
\text { en } \\
\text { final } \\
*\end{array}$ \\
\hline V & $\begin{array}{l}\text { Ley de } \\
\text { Coulo } \\
\mathrm{mb}\end{array}$ & $\begin{array}{l}\text { Campo } \\
\text { s }\end{array}$ & $\begin{array}{l}\text { Circuitos } \\
\text { de }\end{array}$ & $\begin{array}{l}\text { Resisti } \\
\text { vidad }\end{array}$ & $\begin{array}{l}\text { Circuit } \\
\text { os de } \\
\text { c.c }\end{array}$ & $\begin{array}{l}\text { Inducc } \\
\text { ión } \\
\text { electro }\end{array}$ & $\begin{array}{l}\text { Circu } \\
\text { itos } \\
\text { RLC }\end{array}$ & $\begin{array}{l}\text { Exam } \\
\text { en } \\
\text { final }\end{array}$ \\
\hline
\end{tabular}




\begin{tabular}{|c|c|c|c|c|c|c|c|c|}
\hline & & $\begin{array}{l}\text { eléctric } \\
\text { os }\end{array}$ & $\begin{array}{l}\text { condensad } \\
\text { ores }\end{array}$ & $\begin{array}{l}\text { eléctric } \\
\text { a }\end{array}$ & & $\begin{array}{l}\text { magné } \\
\text { tica }\end{array}$ & & $*$ \\
\hline VI & $\begin{array}{l}\text { Ley de } \\
\text { Coulo } \\
\text { mb }\end{array}$ & $\begin{array}{l}\text { Campo } \\
\mathrm{s} \\
\text { eléctric } \\
\text { os }\end{array}$ & $\begin{array}{l}\text { Circuitos } \\
\text { de } \\
\text { condensad } \\
\text { ores }\end{array}$ & $\begin{array}{l}\text { Resisti } \\
\text { vidad } \\
\text { eléctric } \\
\text { a }\end{array}$ & $\begin{array}{l}\text { Circuit } \\
\text { os de } \\
\text { c.c }\end{array}$ & $\begin{array}{l}\text { Inducc } \\
\text { ión } \\
\text { electro } \\
\text { magné } \\
\text { tica }\end{array}$ & $\begin{array}{l}\text { Circu } \\
\text { itos } \\
\text { RLC }\end{array}$ & $\begin{array}{l}\text { Exam } \\
\text { en } \\
\text { final } \\
*\end{array}$ \\
\hline VII & $\begin{array}{l}\text { Ley de } \\
\text { Coulo } \\
\text { mb }\end{array}$ & $\begin{array}{l}\text { Campo } \\
\text { s } \\
\text { eléctric } \\
\text { os }\end{array}$ & $\begin{array}{l}\text { Circuitos } \\
\text { de } \\
\text { condensad } \\
\text { ores }\end{array}$ & $\begin{array}{l}\text { Resisti } \\
\text { vidad } \\
\text { eléctric } \\
\text { a }\end{array}$ & $\begin{array}{l}\text { Circuit } \\
\text { os de } \\
\text { c.c }\end{array}$ & $\begin{array}{l}\text { Inducc } \\
\text { ión } \\
\text { electro } \\
\text { magné } \\
\text { tica }\end{array}$ & $\begin{array}{l}\text { Circu } \\
\text { itos } \\
\text { RLC }\end{array}$ & $\begin{array}{l}\text { Exam } \\
\text { en } \\
\text { final } \\
*\end{array}$ \\
\hline VIII & $\begin{array}{l}\text { Ley de } \\
\text { Coulo } \\
\mathrm{mb}\end{array}$ & $\begin{array}{l}\text { Campo } \\
\text { s } \\
\text { eléctric } \\
\text { os }\end{array}$ & $\begin{array}{l}\text { Circuitos } \\
\text { de } \\
\text { condensad } \\
\text { ores }\end{array}$ & $\begin{array}{l}\text { Resisti } \\
\text { vidad } \\
\text { eléctric } \\
\text { a }\end{array}$ & $\begin{array}{l}\text { Circuit } \\
\text { os de } \\
\text { c.c }\end{array}$ & $\begin{array}{l}\text { Inducc } \\
\text { ión } \\
\text { electro } \\
\text { magné } \\
\text { tica }\end{array}$ & $\begin{array}{l}\text { Circu } \\
\text { itos } \\
\text { RLC }\end{array}$ & $\begin{array}{l}\text { Exam } \\
\text { en } \\
\text { final } \\
*\end{array}$ \\
\hline
\end{tabular}




\section{Objetivos}

Aplicar los métodos de análisis de datos usados en ingeniería para explicar los fenómenos electromagnéticos que ocurren en el mundo real.

Manejar instrumentos de medición eléctrica, haciendo un análisis de los errores que pueden cometerse en la parte experimental.

Evidenciar la importancia de la experimentación, como una forma de comprobar los principios físicos que se aprenden en la clase teórica del curso de electromagnetismo. 


\section{Metodología del curso}

Para el buen desempeño durante las prácticas del laboratorio de física se deben observar las siguientes recomendaciones:

- Durante la práctica, evite mantener dispositivos tales como: teléfonos celulares y demás objetos personales sobre el mesón de trabajo, estos pueden eventualmente convertirse en elementos que desvían su atención y la de sus compañeros. En su mesón de trabajo solamente debe permanecer, su computador y sus herramientas de trabajo. Para colocar su material que no hace parte de la práctica, el laboratorio dispone de un armario metálico ubicado cerca de la puerta. Se recomienda no perturbar el trabajo de los compañeros, evite visitar los mesones vecinos y las visitas de personas ajenas a la sesión del laboratorio.

- El laboratorio requiere de la lectura previa de los temas que se relacionan con la práctica correspondiente según indicaciones presentadas en este manual, como preparación para el quiz de entrada que se realizará en cada sesión.

- Para la toma de datos, los subgrupos se conformarán el primer día de clase y se asignará un número para identificar las prácticas que realizarán durante el semestre sin confusión alguna. La duración de cada experiencia es de 4 horas según las fechas estipuladas en el anexo 2 y consta de aproximadamente 2 horas para la toma de datos y 2 horas para la elaboración del informe.

- El material relacionado con la toma de datos estará ubicado en los mesones correspondientes, el cual dejarán de manera ordenada al terminar su práctica.

- La práctica de laboratorio no tiene derecho a supletorio. En casos excepcionales se debe presentar una excusa, por escrito, dirigida al docente de laboratorio, quien tramitará dicha solicitud ante el equipo docente, para determinar la aceptación o no de dicha excusa. En caso de ser rechazada el estudiante tendrá nota de 0.0 (cero punto cero) en la sesión correspondiente y en caso de ser aceptada, se recuperará la experiencia en una única sesión programada por el equipo docente. 
- El laboratorio aporta el $20 \%$ de la nota de la asignatura de física: $10 \%$ para el primer corte (donde las prácticas de laboratorio serán el 7\% y los quices 3\%); el 10\% restante (correspondiente a las demás prácticas serán el $3 \%$, y los quiches $2 \%$ además de un examen final que aporta un 5\%) para el segundo corte, según las fechas estipuladas por la Universidad.

En caso de inasistencia a una de las prácticas, hay una única sesión (fecha) en que se realizará la práctica pendiente con autorización justificada ante el profesor de laboratorio, avalada por el equipo docente. Esta fecha se indica en el cuadro de fechas. 


\section{Sesión 1- Ley de Coulomb}

- Temas de consulta - Ley de Coulomb (Preparación previa a la práctica)

- Diagramas vectoriales

- Suma gráfica y analítica de vectores

- Ley de coulomb

\section{Competencias}

Al finalizar la práctica el estudiante estará en capacidad de:

Identificar las fuerzas presentes, su configuración y diagrama en el modelo dado.

Cuantificar la magnitud de la fuerza eléctrica resultante para un sistema de cargas puntuales mediante la ley de Coulomb.

Obtener la fuerza eléctrica neta para un sistema de cargas puntuales usando operaciones vectoriales.

\section{Procedimiento}

Suponga que cuatro cargas puntuales $\boldsymbol{q}_{1}, \boldsymbol{q}_{2}, \boldsymbol{q}_{3}$ y $\boldsymbol{q}_{4}$, se colocan en los vértices de un cuadrado de lado a, como se muestra en la figura. Estas cuatro cargas ejercen interacción eléctrica con una quinta carga $\boldsymbol{q}_{0}$ ubicada sobre el punto $\mathrm{p}(\mathrm{x}, \mathrm{y})$.

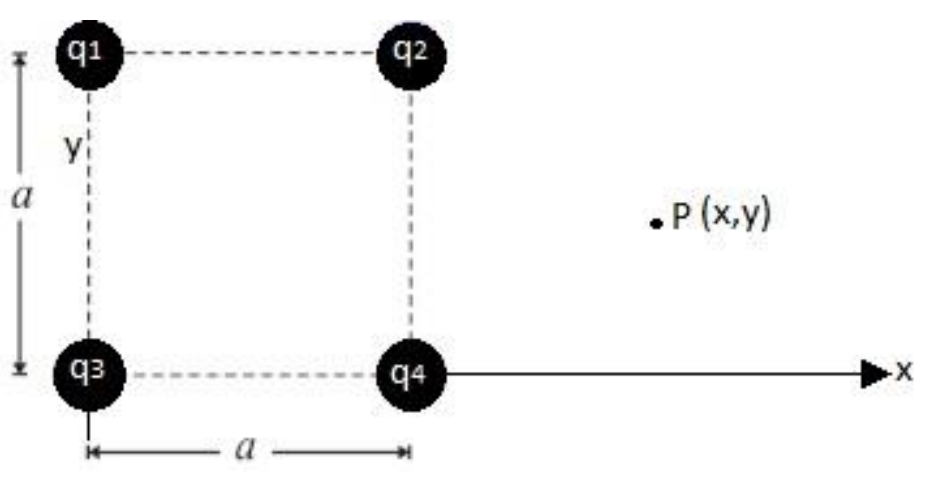


Figura 1. Sistema de cargas puntuales

Con los datos de carga, el valor del lado $\boldsymbol{a}$ y las coordenadas del punto $\boldsymbol{p}$ suministrados por su profesor, complete las columnas 2 y 3 de la siguiente tabla. Para completar las columnas restantes es necesario ir al ítem 1 del apartado Análisis.

Tabla 1

\begin{tabular}{|c|c|c|c|c|c|}
\hline Carga & $\begin{array}{l}\text { Magnitud } \\
\text { de la carga } \\
\text { (c) }\end{array}$ & $\begin{array}{l}\text { Distancia de } \\
\text { la carga al } \\
\text { punto p }(m)\end{array}$ & $\begin{array}{l}\text { Magnitud de la } \\
\text { fuerza eléctrica } \\
\text { en el punto p (n) }\end{array}$ & $\begin{array}{l}\text { Dirección de } \\
\text { la fuerza en p } \\
\text { (ángulo en } \\
\text { grados) }\end{array}$ & $\begin{array}{l}\text { Vector fuerza } \\
\vec{F} \quad(n) . \\
\text { (componentes } \\
\text { vectoriales) }\end{array}$ \\
\hline $\mathrm{Q}_{1}$ & & & & & \\
\hline $\mathrm{Q}_{2}$ & & & & & \\
\hline $\mathrm{Q}_{3}$ & & & & & \\
\hline $\mathrm{Q}_{4}$ & & & & & \\
\hline
\end{tabular}

En un experimento de laboratorio usado para comprobar la ley de Coulomb se toman dos esferas pequeñas idénticas de masa $15 \mathrm{~g}$, que cuelgan de hilos de seda de longitud $\mathrm{l}=60 \mathrm{~cm}$ desde un punto común. Cuando se proporciona a las esferas cantidades iguales de carga $+q$, estas se separan una de la otra, permitiendo que cada hilo haga un ángulo $\theta$ con la vertical, como se muestra en la figura 2. 


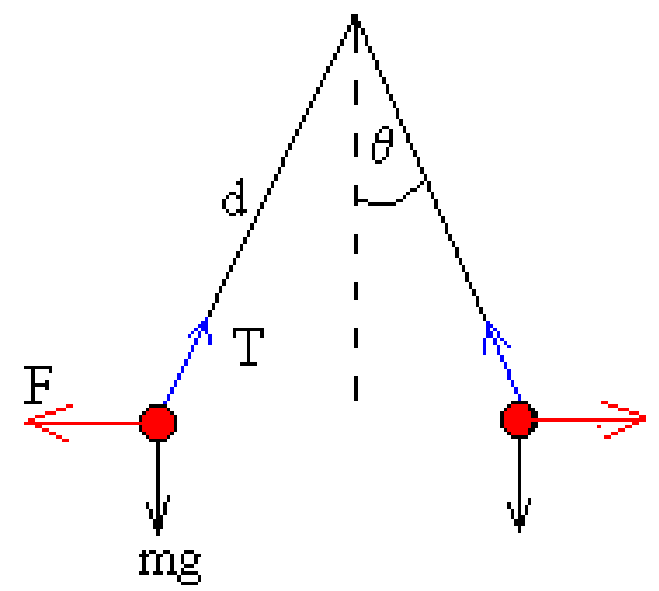

Figura 2. Tomada de sc.ehu.es

Para cada carga proporcionada se mide el ángulo $\theta$ que se desplaza la cuerda. Los datos obtenidos mediante la realización del experimento se muestran en la siguiente tabla:

Tabla 2.

\begin{tabular}{|c|c|c|c|c|c|c|c|c|}
\hline \multicolumn{6}{|c|}{$\Theta($ grados $)$} & \multirow[t]{2}{*}{$Q(\mu c)$} & \multirow[t]{2}{*}{$R(m)$} & \multirow[t]{2}{*}{$F e(n)$} \\
\hline$\Theta_{1}$ & $\boldsymbol{\Theta}_{2}$ & $\boldsymbol{\Theta}_{3}$ & $\boldsymbol{\Theta}_{4}$ & $\Theta_{5}$ & $\boldsymbol{\Theta}_{\text {prom }}$ & & & \\
\hline 16 & 16 & 15 & 16 & 15 & & 9,63 & & \\
\hline 12 & 12 & 11 & 11 & 12 & & 10,9 & & \\
\hline 8 & 8 & 8 & 7 & 7,5 & & 13,36 & & \\
\hline 5 & 5 & 5,5 & 5 & 5 & & 16,22 & & \\
\hline 4 & 3,5 & 3 & 3 & 3 & & 20,3 & & \\
\hline 1,5 & 2 & 1 & 2 & 2 & & 28,3 & & \\
\hline
\end{tabular}

\section{Análisis}

1. Con los datos experimentales de la tabla 1:

1.1 usando la ley de Coulomb, y encontrando las componentes vectoriales de la fuerza eléctrica producida por cada carga sobre las carga $\boldsymbol{q}_{\boldsymbol{0}}$ ubicada en el punto $\mathrm{p}$, halle analíticamente la fuerza 
neta aplicada (magnitud y dirección), sobre $\boldsymbol{q}_{\boldsymbol{0}}$. Repita este procedimiento para dos ubicaciones del punto $\mathrm{p}(\mathrm{x}, \mathrm{y})$, que hayan sido sugeridos por el profesor.

2. Con los datos experimentales de la tabla 2:

2.1 Convierta los datos a unidades del sistema MKS.

2.2 Realice el análisis dinámico del sistema de partículas cargadas para encontrar la fuerza eléctrica y la distancia de separación entre cargas.

2.3 Dibuje la gráfica fuerza (n) vs (carga/distancia $)^{2}$ en $\mathrm{c}^{2} / \mathrm{m}^{2}$

2.4 Para la gráfica obtenida, si es una línea recta, plantee la ecuación que representa dicha función. 2.5 De acuerdo a la ley de coulomb $F=\frac{K Q^{2}}{r^{2}}$, dado que las dos cargas del experimento son iguales, compare la ecuación obtenida experimentalmente con la ecuación teórica de la ley de Coulomb y determine el valor de k. ¿Qué representa físicamente dicha constante?

2.6 Halle el porcentaje de error para la constante k, de acuerdo a su valor teórico comparado con su valor experimental hallado.

3. Elabore conclusiones relacionadas con la fuerza eléctrica, de acuerdo a la posición de la carga q y el efecto de las demás cargas sobre ella. (tenga en cuenta las variaciones con respecto a las distancias de cada carga sobre q, el signo de las cargas y el análisis vectorial).

4. Elabore conclusiones con respecto a las gráficas obtenidas con relación al fenómeno estudiado (ley de Coulomb)

5. Plantee tres aplicaciones relacionadas con sus estudios de ingeniería que se relacionen con el fenómeno físico estudiado en esta sesión. 



\section{Bibliografía}

Libro digital: Sears-Zemansky, et al. Física universitaria con física moderna. Volumen 1. Editorial Pearson Education. Edición 13. Año 2013. Link: http://www.ebooks724.com.aure.unab.edu.co/onlinepdfjs/view.aspx

Serway r., et al. Física para ciencias en ingeniería. Volumen 1. Editorial Cengage Learning. Edición 9. Año 2014

Ohanian h., et al. Física para ingeniería y ciencias. Volumen 1. Editorial McGraw-Hillinteramericana. Año 2009. 


\section{Sesión 2- Campos Eléctricos}

- Temas de consulta - Campos Eléctricos (Preparación previa a la práctica)

- Intensidad del campo eléctrico

- Campo eléctrico debido a distribuciones de carga continua.

- Líneas de fuerza.

- Superficies equipotenciales.

\section{Competencias}

Al finalizar la práctica el estudiante estará en capacidad de:

Identificar las líneas de fuerza producidas por diferentes distribuciones de carga.

Graficar las superficies equipotenciales para diferentes puntos entre las superficies cargadas.

Comparar ideas y opiniones para la toma de decisiones y planes respecto a la temática estudiada junto con sus compañeros de grupo.

\section{Materiales}

Cubetas

Electrodos

Puntas de multímetro o sondas móvil y fija

Multímetros.

Solución salina o gel eléctricamente conductor

Plantilla de papel milimetrado.

\section{Procedimiento}


Con los materiales mostrados en la figura (1a), realice el montaje de la figura (1b).

Nota: revise con el profesor las conexiones antes de tomar los datos.

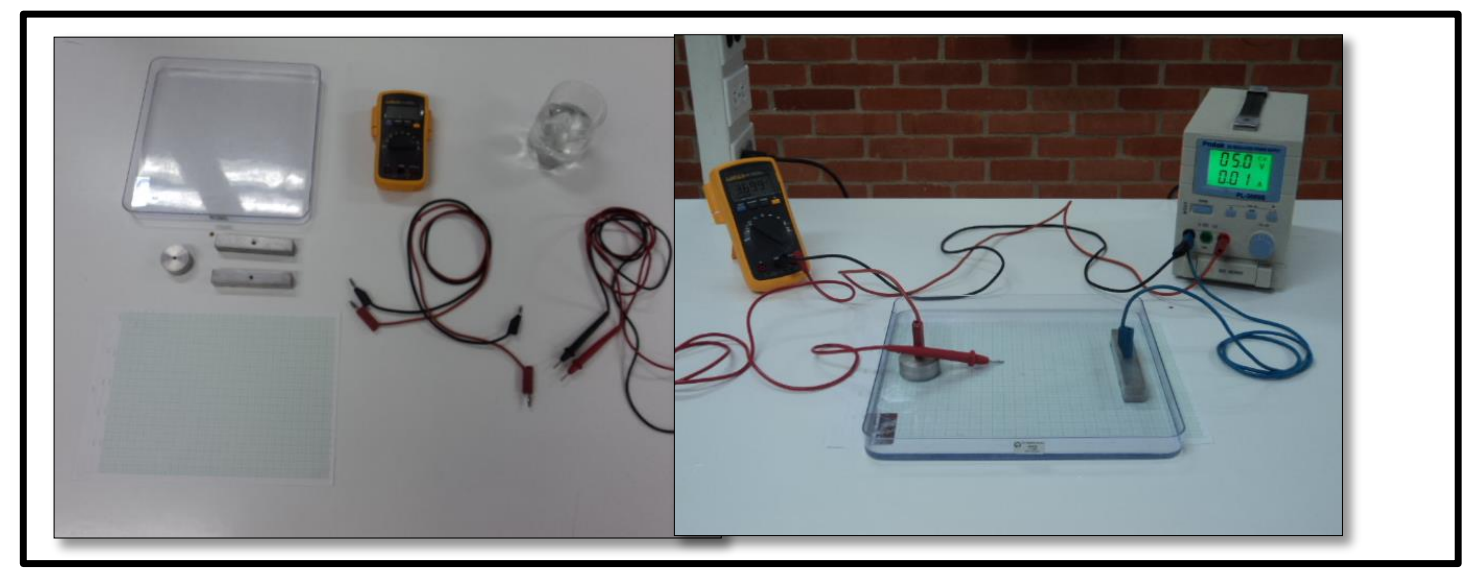

Figura 1a. Materiales utilizados

figura $1 \mathrm{~b}$. Montaje del experimento

Disponga de una cubeta con un pequeño volumen de solución salina o gel eléctricamente conductor o agua clorada, como lo muestra la figura 1. En la parte inferior de la cubeta coloque la plantilla de papel milimetrado en la que previamente ha asignado las coordenadas rectangulares.

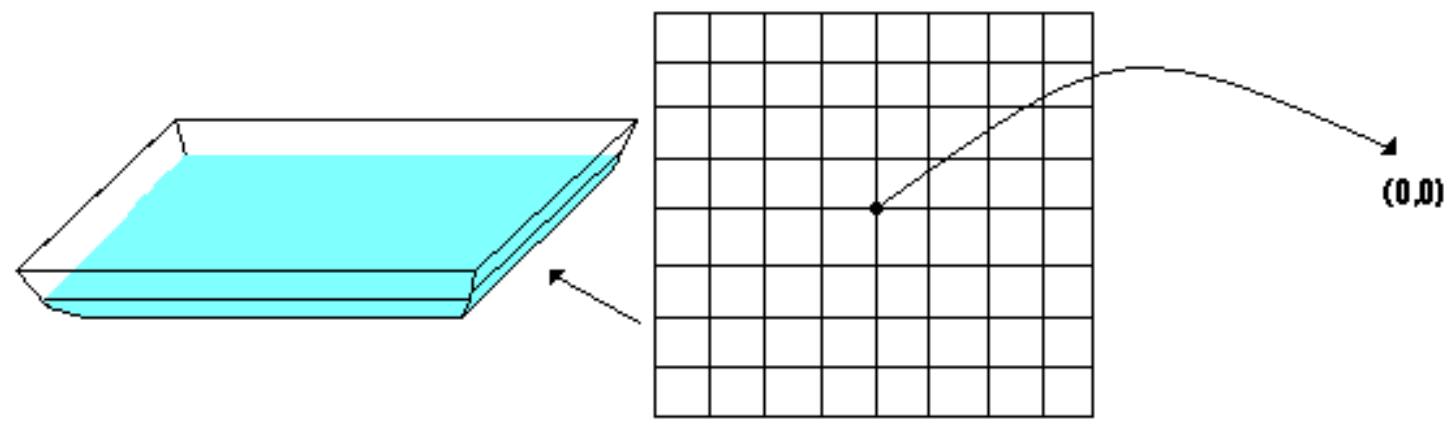

Figura 2. Cubeta con solución salina 
Conecte un par de electrodos con igual o diferente geometría a una fuente de voltaje, suministre un valor de tensión (voltaje), el cual será proporcionado por el profesor. Los electrodos conectados a la fuente constituyen distribuciones de carga (ver figura 2).

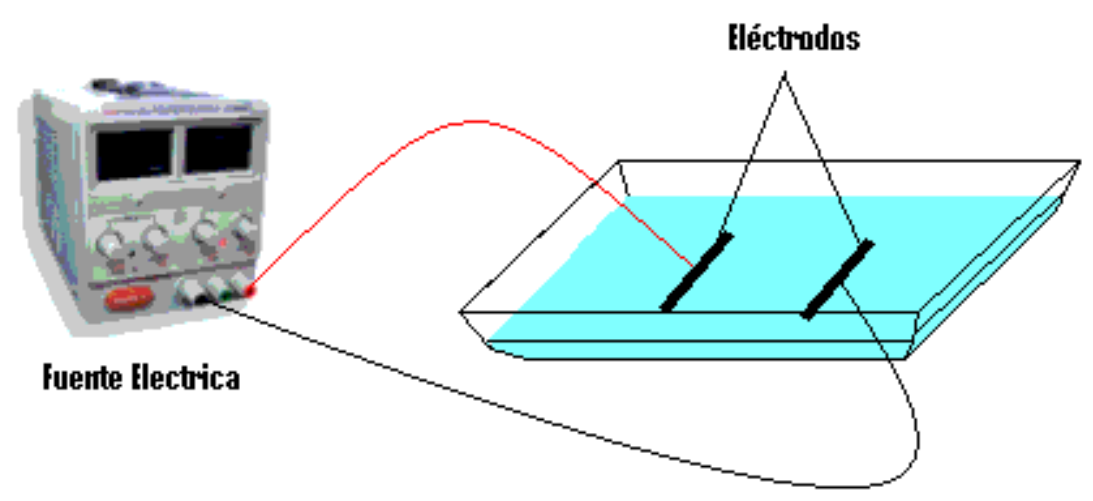

Figura 3. Disposición de los electrodos en la cubeta

Para identificar las curvas equipotenciales en el plano de la cubeta necesitamos un galvanómetro o un voltímetro. Ubique y mantenga fija en cualquier punto de la cubeta una de las sondas del voltímetro, la otra sonda se desplazará por diferentes puntos de la cubeta (figura 3). En la hoja de papel milimetrado, diferente de la que yace debajo de la cubeta, anote los puntos para los cuales se presenta la misma lectura de voltaje en el multímetro. Para obtener un buen perfil de las curvas equipotenciales es necesario que tome medidas de 4 voltajes en los cuadrantes 1 y 4 , y otros 4 voltajes en los cuadrantes 2 y 3 . Para cada curva de voltaje debe buscar al menos 8 mediciones. 


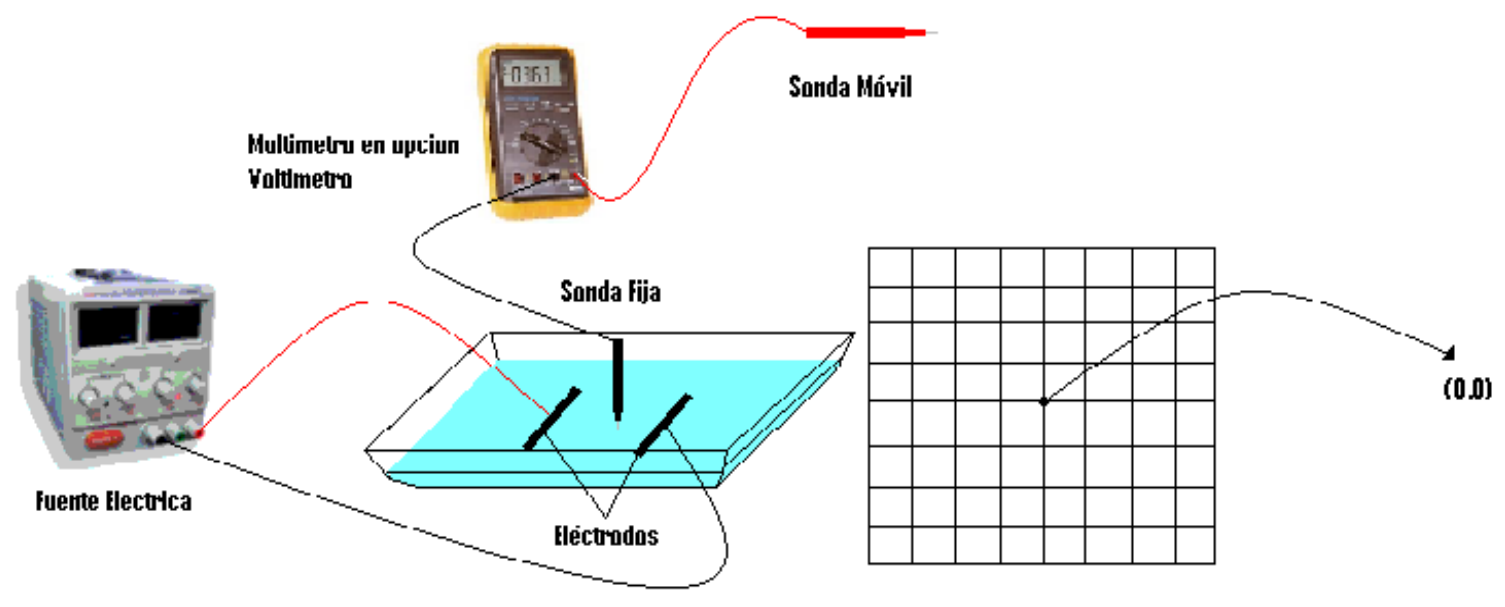

Figura 4. Identificación de curvas equipotenciales.

\section{Análisis}

1. Con base en los datos obtenidos en el punto tres del procedimiento, para las diferentes configuraciones de electrodos, trace varias curvas equipotenciales uniendo los puntos de igual valor de diferencia de potencial. (el número de curvas será sugerido por el profesor).

2. Trace las líneas de campo de acuerdo a las curvas equipotenciales identificadas en el punto anterior.

3. Dibuje el vector intensidad de campo eléctrico, en diferentes puntos, sobre el diagrama de líneas de campo.

4. Exprese sus observaciones y conclusiones sobre los aspectos físicos de su experimento teniendo en cuenta: la teoría, el procedimiento, la tabla de datos, las gráficas y las fuentes de error.

5. Plantee tres aplicaciones relacionadas con sus estudios de ingeniería que se relacionen con el fenómeno físico estudiado en esta sesión. 



\section{Bibliografía}

Libro digital: Sears-Zemansky, et al. Física universitaria con física moderna. Volumen 2. Editorial Pearson Education. Edición 13. Año 2013. Link: http://www.ebooks724.com.aure.unab.edu.co/onlinepdfjs/view.aspx.

Serway r., et al. Física para ciencias en ingeniería. Volumen 2. Editorial Cengage Learning. Edición 9. Año 2014.

Ohanian h., et al. Física para ingeniería y ciencias. Volumen 2. Editorial McGraw-Hillinteramericana. Año 2009. 


\section{Sesión 3- Circuitos de Condensadores}

- Temas de consulta - Circuitos de Condensadores (Preparación previa a la práctica)

- Concepto de capacitancia

- Capacitancia equivalente circuitos serie y paralelo en condensadores

- Capacitancia equivalente circuitos mixtos en condensadores

- Capacitancia carga y voltaje en condensadores

\section{Competencias}

Al finalizar la práctica el estudiante estará en capacidad de:

Caracterizar diferentes configuraciones (serie, paralelo y mixta) que se pueden lograr con condensadores.

Calcular la capacitancia equivalente para cada una de las configuraciones indicadas.

Comparar ideas y opiniones para la toma de decisiones y planes respecto a la temática estudiada junto con sus compañeros de grupo.

\section{Materiales}

Protoboard (tableta de experimentación)

Condensadores de diferente valor de capacitancia

Multímetro (voltímetro y amperímetro)

Fuente de corriente continua

Cables

Puentes de conexión 


\section{Procedimiento}

Con los materiales suministrados (ver figura 1). Realice los montajes correspondientes a los circuitos serie, paralelo y mixto.

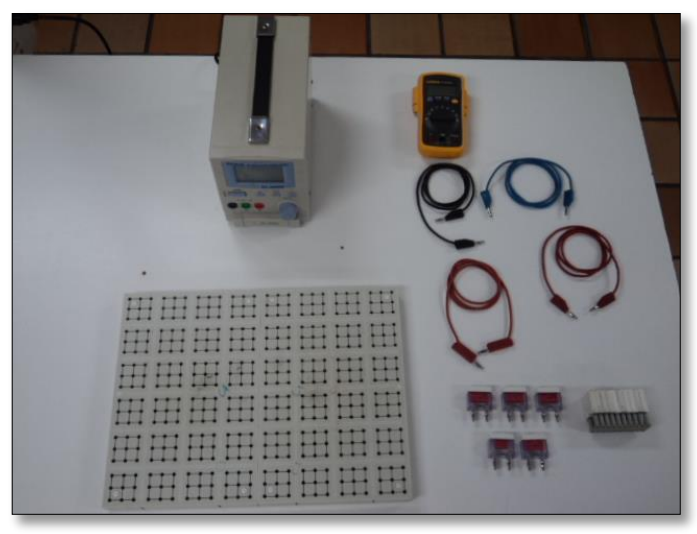

Figura 1. Materiales usados en el experimento

Inicialmente mida la capacitancia de cada condensador sin incluir en el circuito corriente aún; registre los valores en las tablas y recuerde el orden de cada condensador para ubicarlos en los demás circuitos.

Realice cada montaje sugerido a continuación y mida el valor de la diferencia de potencial (v) de cada condensador; para cada uno de los circuitos sugeridos a continuación.

\section{Circuito en serie:}




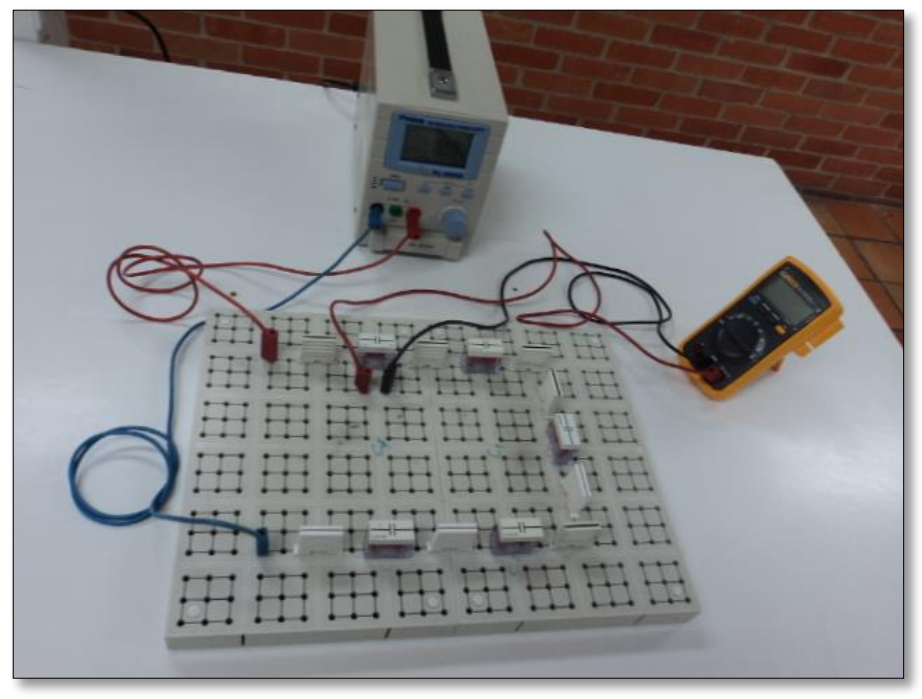

Figura 2. Montaje circuito en serie de condensadores

Nota: revise con el profesor las conexiones antes de tomar datos. El valor del voltaje será sugerido por el profesor.

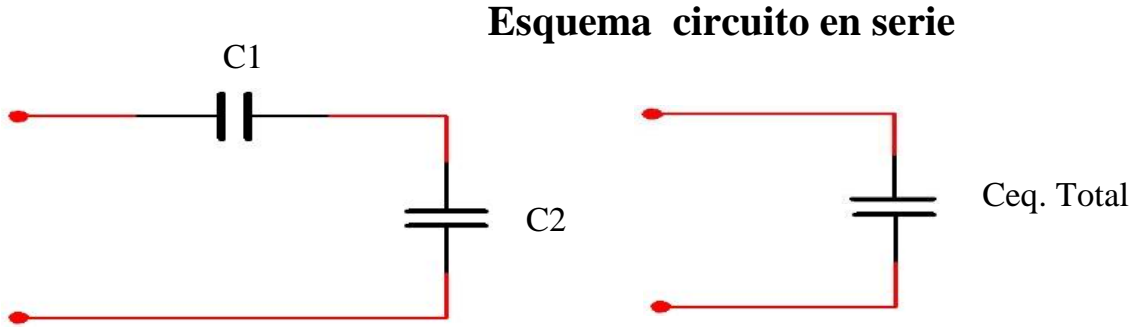

\begin{tabular}{|l|l|l|l|}
\hline & Capacitancia $(\mu f)$ & Carga $(\mu c)$ & $\begin{array}{l}\text { Diferencia } \\
\text { potencial }(v)\end{array}$ \\
\hline $\mathrm{C}_{1}$ & & & \\
\hline $\mathrm{C}_{2}$ & & & \\
\hline
\end{tabular}




\begin{tabular}{|l|l|l|l|}
\hline $\mathrm{C}_{3}$ & & & \\
\hline $\mathrm{C}_{4}$ & & & \\
\hline $\mathrm{C}_{5}$ & & & \\
\hline $\mathrm{C}_{\text {equivalente }}$ & & & \\
\hline
\end{tabular}

\section{Circuito en paralelo}

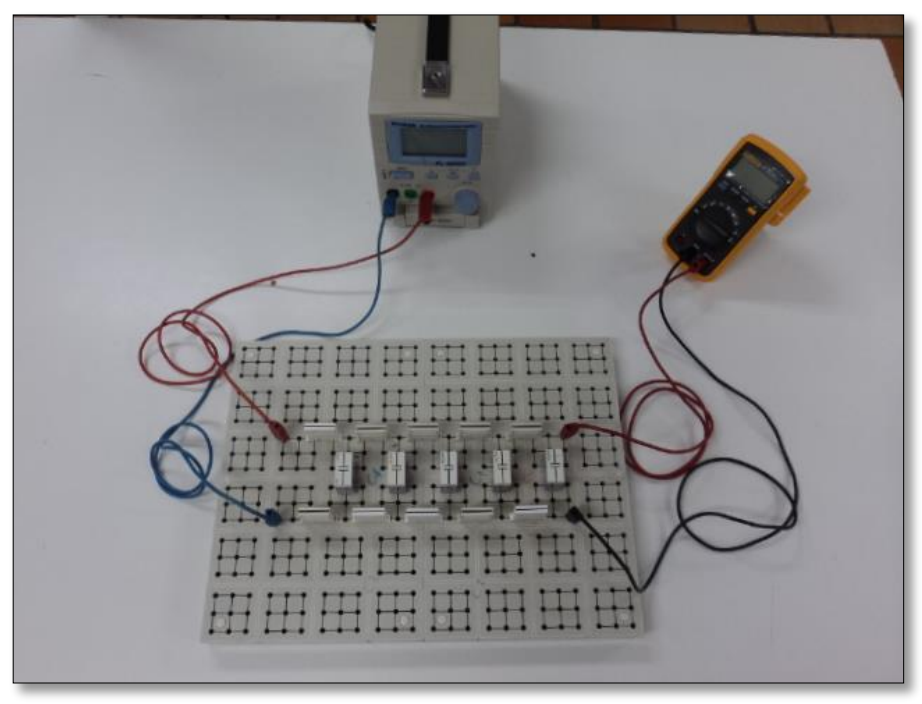

Figura 3. Montaje circuito en paralelo de condensadores

Esquema de circuito de condensadores en paralelo

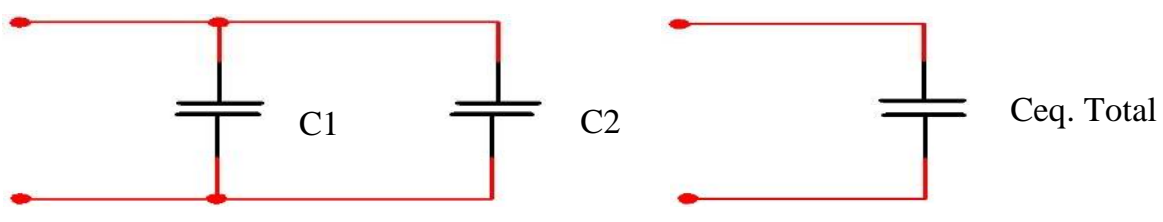

\begin{tabular}{|l|l|l|l|}
\hline & Capacitancia $(\mu f)$ & Carga $(\mu c)$ & $\begin{array}{l}\text { Diferencia } \\
\text { potencial }(v)\end{array}$
\end{tabular}




\begin{tabular}{|l|l|l|l|}
\hline $\mathbf{C}_{1}$ & & & \\
\hline $\mathbf{C}_{2}$ & & & \\
\hline $\mathrm{C}_{3}$ & & & \\
\hline $\mathrm{C}_{4}$ & & & \\
\hline $\mathrm{C}_{5}$ & & & \\
\hline $\mathrm{C}_{\text {equivalente }}$ & & & \\
\hline
\end{tabular}

\section{Circuito mixto}

Realice la siguiente configuración de condensadores para este circuito, de acuerdo al esquema mostrado a continuación.
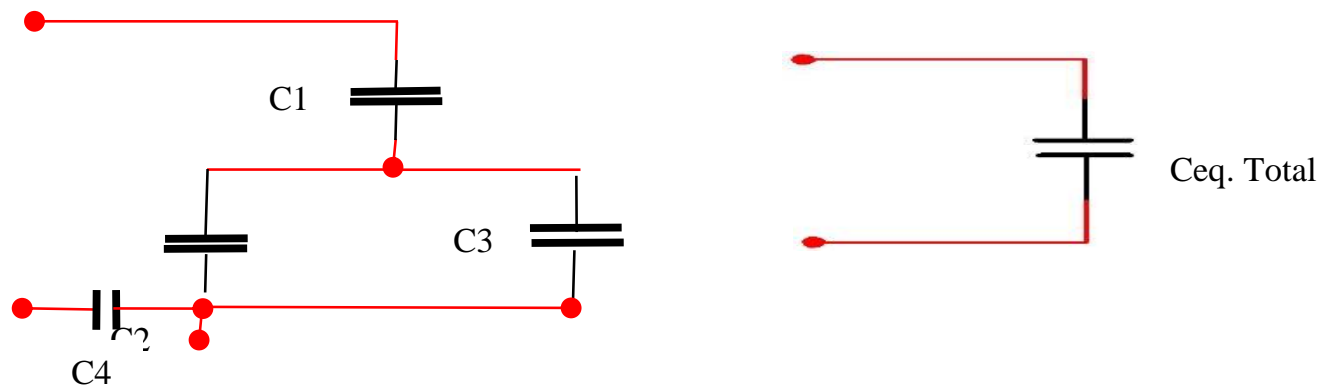

\section{Circuito mixto}

\begin{tabular}{|l|l|l|l|}
\hline & Capacitancia $(\mu f)$ & Carga $(\mu c)$ & $\begin{array}{l}\text { Diferencia de potencial } \\
(v)\end{array}$ \\
\hline $\mathrm{C}_{1}$ & & & \\
\hline $\mathrm{C}_{2}$ & & & \\
\hline
\end{tabular}




\begin{tabular}{|l|l|l|l|}
\hline $\mathbf{C}_{3}$ & & & \\
\hline $\mathbf{C}_{4}$ & & & \\
\hline $\mathbf{C}_{5}$ & & & \\
\hline $\mathbf{C}_{\text {equivalente }}$ & & & \\
\hline
\end{tabular}

\section{Análisis}

1. Para los circuitos serie y paralelo:

1.1 calcule el valor de la carga en cada condensador a partir de los valores experimentales de la diferencia de potencial y los valores correspondientes de capacitancia de cada condensador.

1.2 calcule la capacitancia equivalente.

1.3 calcule la carga total equivalente.

2. Para el circuito mixto:

2.1 Realice los esquemas de los circuitos hasta reducir a uno solo que represente el circuito total.

2.2 A partir de los valores de diferencia de potencial, y el valor experimental de la capacitancia equivalente de todo el circuito, calcule la diferencia de potencial y la carga en el condensador.

2.3 Calcule el porcentaje de error entre los valores calculados y los datos experimentales para la carga y para la diferencia de potencial total.

2.4 Exprese sus observaciones y conclusiones.

3. Plantee tres aplicaciones relacionadas con sus estudios de ingeniería que se relacionen con el fenómeno físico estudiado en esta sesión. 



\section{Bibliografía}

Ohanian Hans c., Markert John t. Física para ingeniería y ciencias. Vol. 2 mc. Graw-Hill. Tercera edición. México, 2009.

Sears et al. Física universitaria. Vol. 2. Pearson - Addison Wesley, décimo segunda edición. México. 2009.

Serway. Jewet. Física para ciencias e ingeniería vol. 2. Thomson, sexta edición. México. 2005. 


\section{Sesión 4- Resistividad Eléctrica}

- Temas de consulta - Resistividad Eléctrica (Preparación previa a la práctica)

- Resistividad eléctrica.

- Conductividad y conductancia eléctrica.

- Resistencia y ley de Ohm.

- Densidad de corriente, definición microscópica de la ley de Ohm.

\section{Competencias}

Al finalizar la práctica el estudiante estará en capacidad de:

Determinar la resistividad eléctrica $\rho$ de algunos materiales óhmicos.

Verificar experimentalmente la expresión $R=\rho \frac{L}{A}$ para estos materiales.

Comparar ideas y opiniones para la toma de decisiones y planes respecto a la temática estudiada junto con sus compañeros de grupo.

\section{Materiales}

2 multímetros

6 cables

Pinzas o caimanes

Fuente corriente continua

Regla

Alambres de diferentes materiales y diferentes diámetros (Cromo-Níquel, Constantán, Messin o Latón)

Reóstato 


\section{Procedimiento}

Con los materiales mostrados en la figura 1a, realice el montaje de la figura $1 \mathrm{~b}$.

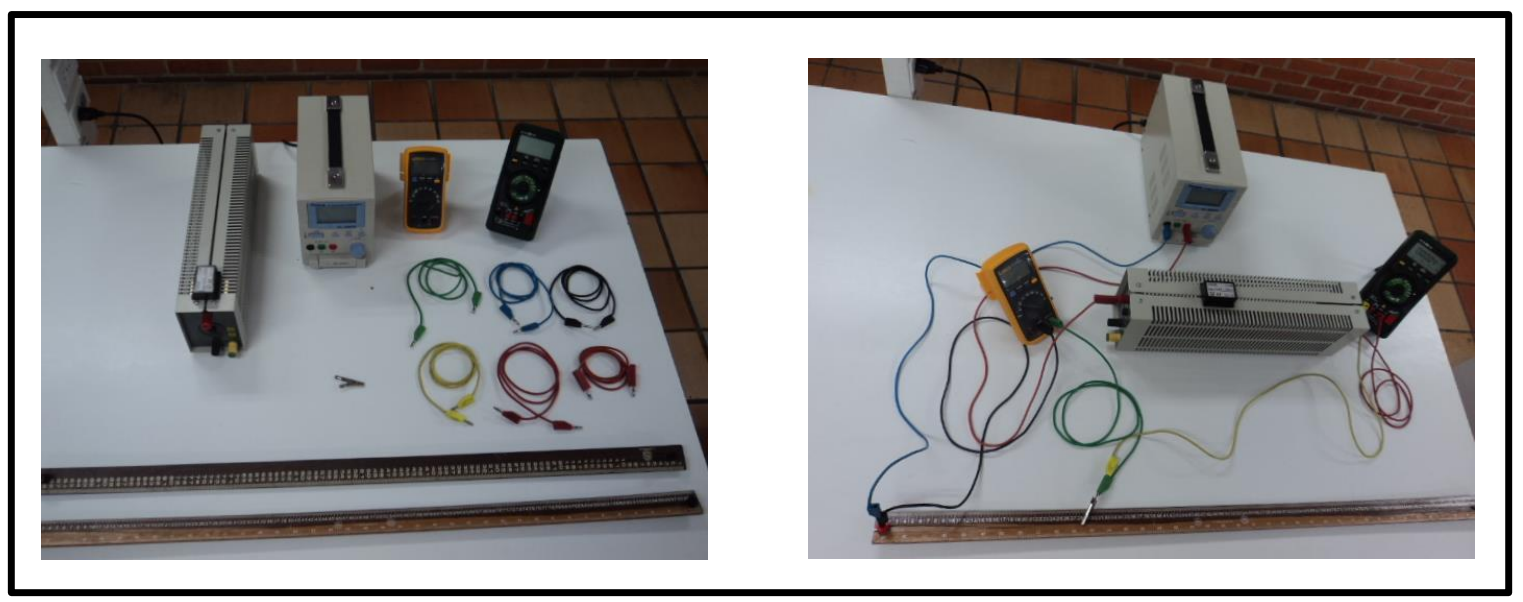

Figura 1a. Materiales usados en el experimento figura 1b. Montaje experimental

Expresión $R=\rho \frac{L}{A}$ para hallar la resistividad de los materiales.

Nota: revise con el profesor las conexiones antes de tomar los datos.

Inicialmente determinaremos la resistividad de los materiales proporcionados por el profesor midiendo directamente, mediante el óhmetro, la resistencia, $\boldsymbol{r}$ de $\boldsymbol{n}$ segmentos de longitud $\boldsymbol{l}$ y secciones transversales constante, $\boldsymbol{a}$.

La figura 2, nos muestra la disposición de los elementos para medir la resistencia directamente 


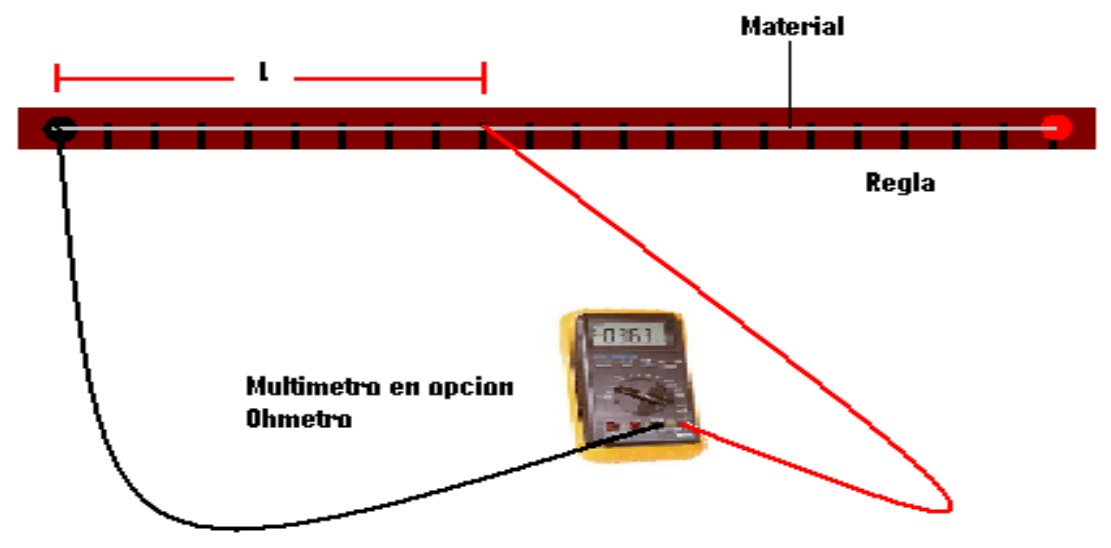

Figura 2. Medida directa de la resistencia de diferentes segmentos de longitud $\boldsymbol{l}$.

Registre los valores de resistencia y longitud de los segmentos en la tabla 1.

La siguiente parte de la experiencia comprende la medida directa de la resistencia mediante la ley de ohm, para esto es necesario registrar la corriente, $\boldsymbol{i}$ y la diferencia de potencial, $\boldsymbol{v}$, a través de cada uno de los $\boldsymbol{n}$ segmentos de longitud $\boldsymbol{l}$. (puede seleccionar los mismos segmentos del punto anterior).

La figura 3. Muestra el montaje para el registro de la resistencia de un segmento de material mediante la ley de Ohm. Se trata de un circuito simple que consta de una fuente de $\varepsilon$ desconocida. Las diferentes porciones del material son seleccionadas desplazando el terminal móvil del circuito de protección. 


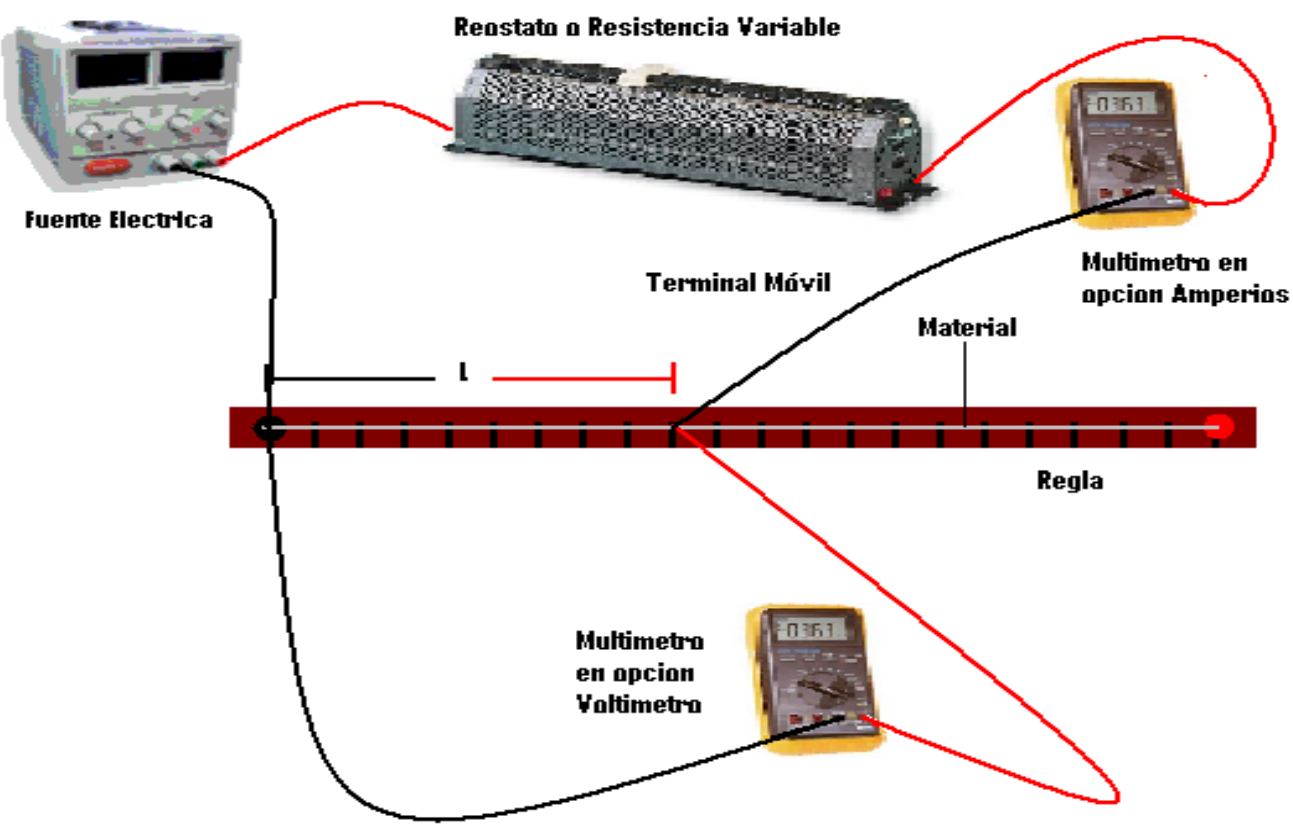

Figura 3. Medida indirecta de la resistencia de segmentos de longitud $\boldsymbol{l}$.

Registre los valores de resistencia y longitud de los segmentos en la tabla 1. 


\begin{tabular}{|c|c|c|c|c|c|c|c|c|c|}
\hline \multicolumn{7}{|c|}{ Tabla 1. Medida directa e indirecta de la resistencia del material: } & \multicolumn{3}{|c|}{ Diámetro $^{\phi}: \quad$ área: } \\
\hline $\begin{array}{l}\text { Longitud } \\
\text { (m) }\end{array}$ & Voltaje (v) & $\begin{array}{l}\text { Corriente } \\
\text { (i) }\end{array}$ & $\begin{array}{l}\text { Resistenci } \\
\text { a } \\
(\Omega)\end{array}$ & $\begin{array}{l}\text { Resistenci } \\
\text { a indirecta } \\
(\Omega)\end{array}$ & $\begin{array}{l}\text { Resistivid } \\
\text { ad directa } \\
(\rho)\end{array}$ & $\begin{array}{l}\text { Resistivid } \\
\text { ad } \\
\text { indirecta } \\
(\rho)\end{array}$ & $\begin{array}{l}\text { Error } \\
\text { resistividad } \\
\text { directa (\%) }\end{array}$ & $\begin{array}{l}\text { Error } \\
\text { resistivida } \\
\text { dindirecta } \\
(\%)\end{array}$ & $\begin{array}{l}\text { Conductiv } \\
\text { idad }(\sigma)\end{array}$ \\
\hline & & & & & & & & & \\
\hline & & & & & & & & & \\
\hline & & & & & & & & & \\
\hline & & & & & & & & & \\
\hline & & & & & & & & & \\
\hline & & & & & & & & & \\
\hline & & & & & & & & & \\
\hline & & & & & & & & & \\
\hline & & & & & & & & & \\
\hline & & & & & & & & & \\
\hline
\end{tabular}




\section{Análisis}

Para cada uno de las muestras suministradas:

Grafique $\boldsymbol{l}$ vs. $\boldsymbol{R}$ directo de acuerdo a los registros de la tabla 1. Interprete físicamente la pendiente y a partir de su valor encuentre la resistividad del material.

Calcule $\boldsymbol{r}$ para los $\boldsymbol{n}$ segmentos del material utilizando la relación de la ley de Ohm.

Grafique $\boldsymbol{l}$ vs. $\boldsymbol{R}$ indirecto de acuerdo a los registros de la tabla 1. Interprete físicamente la pendiente y a partir de su valor encuentre la resistividad del material.

Calcule el porcentaje de error de los valores obtenidos de resistividad eléctrica, para ambos métodos, con los valores reportados en la literatura.

Tabla de resistividad eléctrica teórica de los elementos reportados en la literatura a $20^{\circ} \mathrm{c}$.

\begin{tabular}{|l|l|}
\hline Material & pteórica $\left(\boldsymbol{x} \mathbf{1 0}^{-8} \boldsymbol{\Omega m}\right)$ \\
\hline Constantán & 49 \\
\hline Cromo Níquel o niCromo & 100 \\
\hline Messín o Latón & 7,8 \\
\hline
\end{tabular}

Exprese sus observaciones y conclusiones sobre los aspectos físicos de su experimento teniendo en cuenta: la teoría, el procedimiento, la tabla de datos, las gráficas y las fuentes de error.

Plantee tres aplicaciones relacionadas con sus estudios de ingeniería que se relacionen con el fenómeno físico estudiado en esta sesión. 


\section{Bibliografía}

Ohanian Hans c., Markert John t. Física para ingeniería y ciencias. Vol. 2 mc. Graw-Hill. Tercera edición. México, 2009.

Sears et al. Física universitaria. Vol. 2. Pearson - Addison Wesley, décimo segunda edición. México. 2009.

Serway. Jewet. Física para ciencias e ingeniería vol. 2. Thomson, sexta edición. México. 2005. 


\section{Sesión 5- Circuitos de Corriente Continua}

- Temas de consulta - Circuitos de Corriente Continua (Preparación previa a la práctica)

- Corriente eléctrica.

- Ley de Ohm.

- Reglas de Kirchhoff.

\section{Competencias}

Al finalizar la práctica el estudiante estará en capacidad de:

Verificar el comportamiento de la caída de potencial en los resistores en un circuito de corriente continua.

Aplicar las reglas de Kirchhoff para la solución de circuitos que no se pueden reducir a una configuración simple.

Comparar ideas y opiniones para la toma de decisiones y planes respecto a la temática estudiada junto con sus compañeros de grupo.

\section{Materiales}

Protoboard

Resistencias de diferente valor óhmico pero de la misma potencia.

Multímetro (voltímetro y amperímetro)

Fuente de corriente continua

Cables

Puentes de conexión

\section{Procedimiento}


Para cada uno de los resistores suministrados por el profesor determine el rango seguro de voltaje, $v$ para evitar que el resistor se funda. Utilice la expresión:

$$
V=\sqrt{(R * P)}
$$

Donde $\boldsymbol{r}$ y $\boldsymbol{p}$ son el valor de la resistencia y la potencia nominal disipada del resistor.

Con los elementos suministrados para la práctica mostrados en la figura 1, construya los siguientes circuitos. Nota: el valor del voltaje será sugerido por el profesor. Revise con él las conexiones antes de tomar datos.

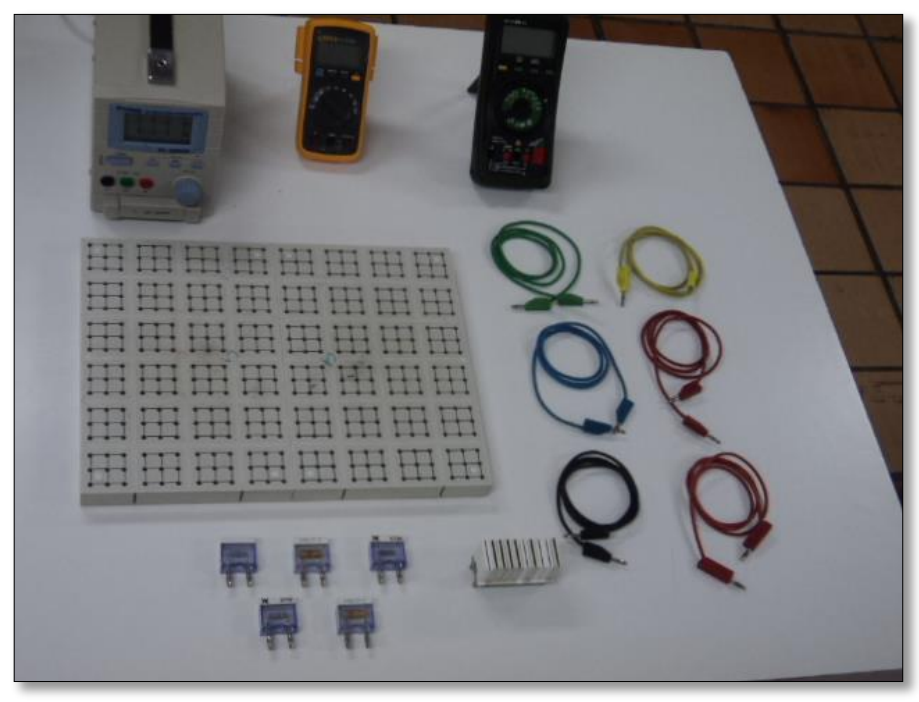

Figura 1. Materiales usados en el experimento

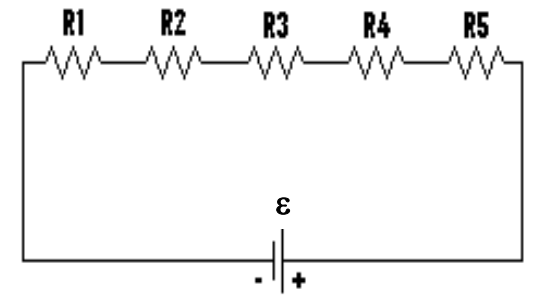

A

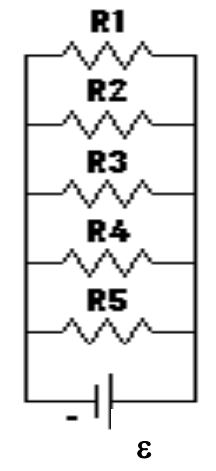

B

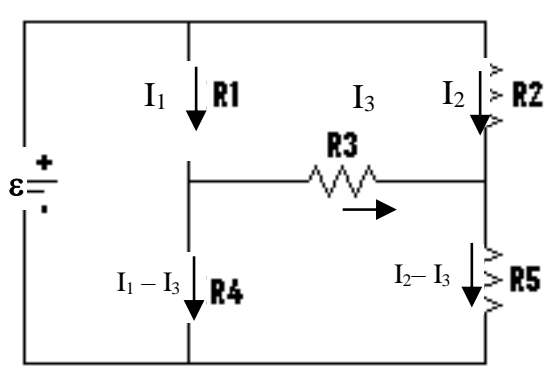

C 
Figura 2. Circuitos resistivos para verificar la ley de Ohm y las reglas de Kirchhoff (serie, paralelo y mixto)

Mida la diferencia de potencial mediante el voltímetro y la corriente con el amperímetro en cada uno de los resistores de los circuitos antes mencionados. Llene la tabla propuesta para cada circuito y registre en ella sus datos.

\section{Circuito en serie}

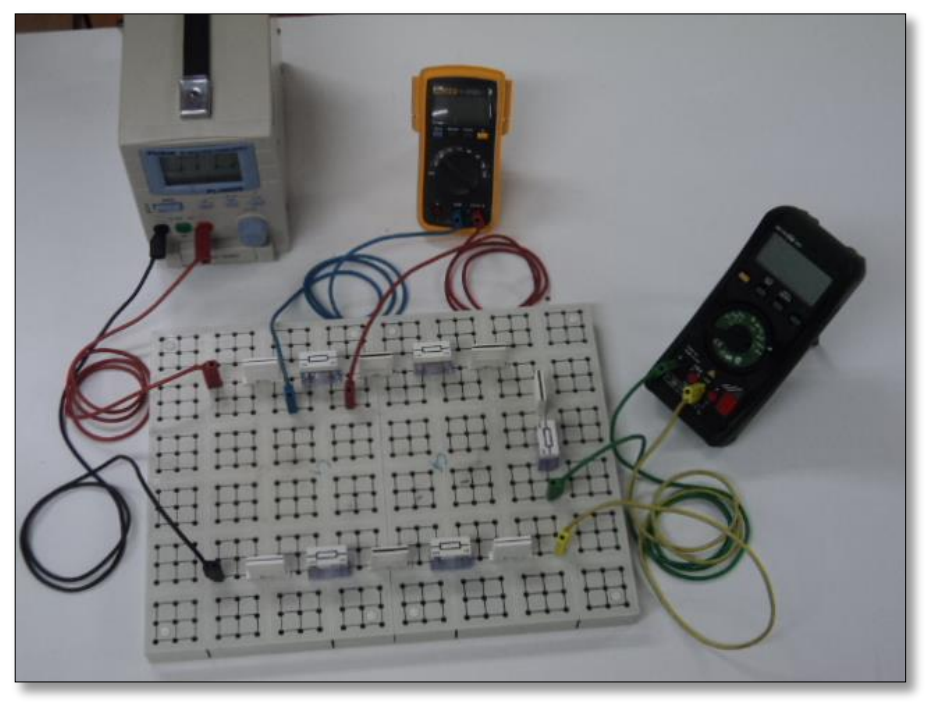

Figura 3. Circuito en serie

\begin{tabular}{|l|l|l|l|l|}
\hline & $\begin{array}{l}\text { Voltaje } \\
\text { Máximo }\end{array}$ & Resistencia $(\Omega)$ & Corriente $(\mathrm{mA})$ & $\begin{array}{l}\text { Diferencia de } \\
\text { potencial (v) }\end{array}$ \\
\hline $\mathbf{R}_{1}$ & & & & \\
\hline $\mathbf{R}_{2}$ & & & & \\
\hline $\mathbf{R}_{3}$ & & & & \\
\hline $\mathbf{R}_{4}$ & & & & \\
\hline $\mathbf{R}_{5}$ & & & & \\
\hline $\mathbf{R}_{\text {equivalente }}$ & & & \\
\hline
\end{tabular}




\section{Circuito en paralelo}

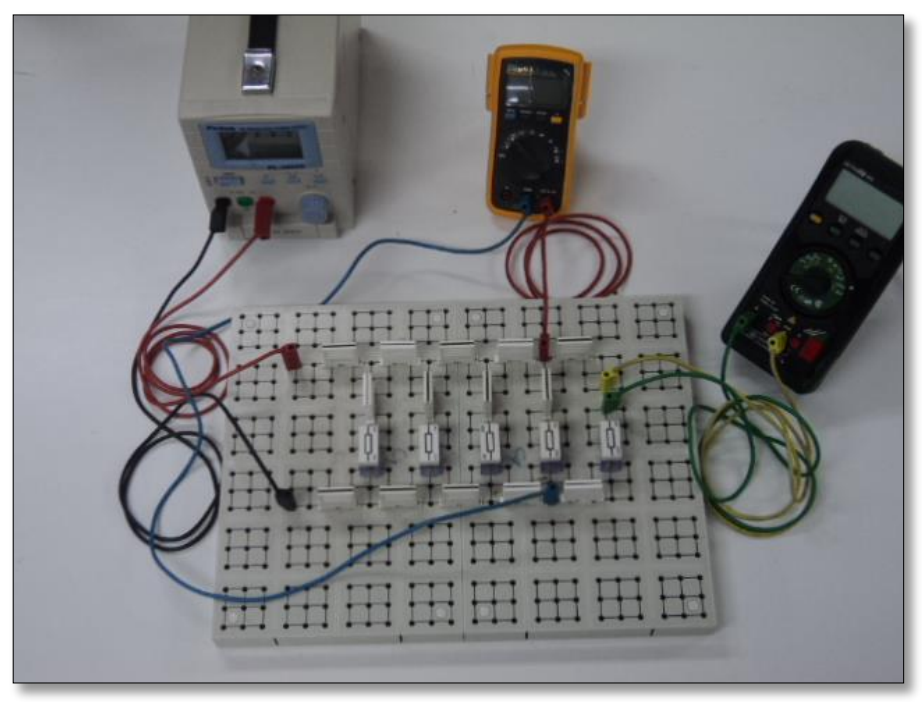

Figura 4. Circuito en paralelo

\begin{tabular}{|l|l|l|l|l|}
\hline & $\begin{array}{c}\text { Voltaje } \\
\text { máximo }\end{array}$ & Resistencia $(\Omega)$ & Corriente (ma) & $\begin{array}{l}\text { Diferencia de } \\
\text { potencial (v) }\end{array}$ \\
\hline $\mathbf{R}_{1}$ & & & & \\
\hline $\mathbf{R}_{2}$ & & & & \\
\hline $\mathbf{R}_{3}$ & & & & \\
\hline $\mathbf{R}_{4}$ & & & & \\
\hline $\mathbf{R}_{5}$ & & & & \\
\hline $\mathbf{R}_{\text {equivalente }}$ & & & \\
\hline
\end{tabular}

Circuito mixto para ley de ohm y reglas de Kirchhoff 


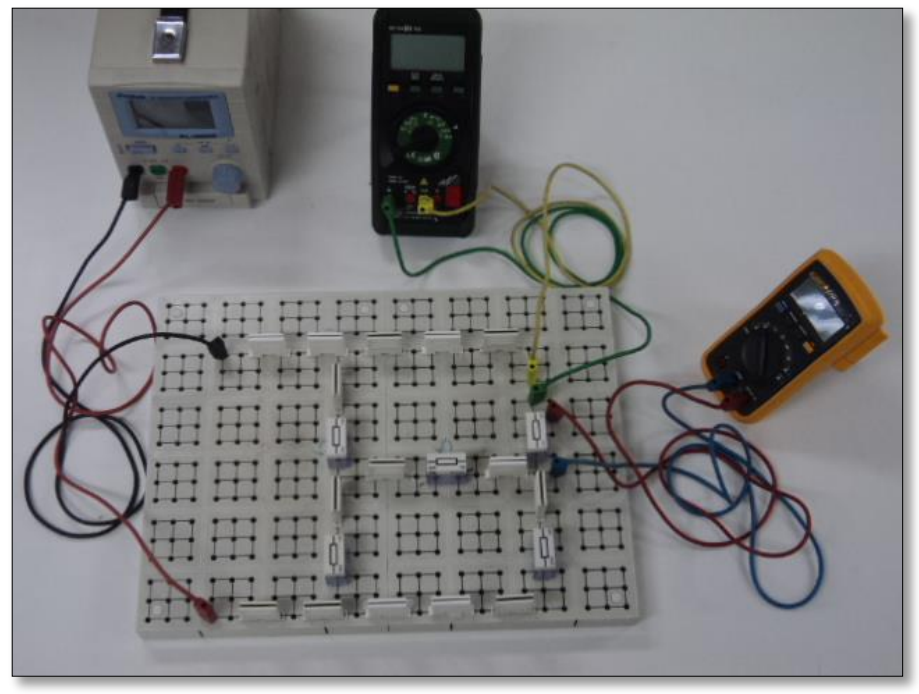

Figura 5. Circuito mixto

\begin{tabular}{|l|l|l|l|l|l|}
\hline & $\begin{array}{l}\text { Resistencia } \\
(\Omega)\end{array}$ & $\begin{array}{l}\text { Corriente } \\
(\mathrm{ma})\end{array}$ & $\begin{array}{l}\text { Corriente } \\
(\mathrm{ma}) \text { exp. }\end{array}$ & \% error & $\begin{array}{l}\text { Diferencia de } \\
\text { potencial (v) }\end{array}$ \\
\hline $\mathbf{R}_{1}$ & & & & & \\
\hline $\mathbf{R}_{2}$ & & & & & \\
\hline $\mathbf{R}_{3}$ & & & & & \\
\hline $\mathbf{R}_{4}$ & & & & & \\
\hline $\mathbf{R}_{5}$ & & & & & \\
\hline $\mathbf{R}_{\text {eq. }}$ & & & & & \\
\hline
\end{tabular}

\section{Análisis}

Utilizando el valor de la corriente en el circuito (a), calcule la diferencia de potencial en cada uno de los resistores. Compare este valor medido. Tabule sus resultados.

En el circuito (b) emplee el valor de la diferencia de potencial y calcule el valor de la corriente en cada resistor. Compare los valores calculados y los experimentales. 
Mediante el empleo de las reglas de Kirchhoff, calcule la corriente y la diferencia de potencial en cada uno de los resistores del circuito (c). Tabule sus resultados.

Calcule el porcentaje de error entre los valores calculados y los datos experimentales.

Exprese sus observaciones y conclusiones sobre los aspectos físicos de su experimento teniendo en cuenta: la teoría, el procedimiento, la tabla de datos, las gráficas y las fuentes de error.

Plantee tres aplicaciones relacionadas con sus estudios de ingeniería que se relacionen con el fenómeno físico estudiado en esta sesión. 


\section{Bibliografía}

Ohanian Hans c., Markert John t. Física para ingeniería y ciencias. Vol. 2 mc. Graw-Hill. Tercera edición. México, 2009.

Sears et al. Física universitaria. Vol. 2. Pearson - Addison Wesley, décimo segunda edición. México. 2009.

Serway. Jewet. Física para ciencias e ingeniería vol. 2. Thomson, sexta edición. México. 2005. 


\section{Sesión 6- Inducción Electromagnética}

Temas de consulta - Inducción Electromagnética (Preparación previa a la práctica)

- Ley de Ampere.

- Ley de Faraday - ley de Lenz.

- Campo magnético en un solenoide.

\section{Competencias}

Al finalizar la práctica, el estudiante estará en capacidad de:

Evidenciar el fenómeno de inducción electromagnética de acuerdo a la ley de Faraday-Lenz para diferentes clases de bobinas.

Comparar ideas y opiniones para la toma de decisiones y planes respecto a la temática estudiada junto con sus compañeros de grupo.

\section{Materiales}

Imanes

Bobinas

Cables

Galvanómetro

Fuentes de corriente continua

Fuente de corriente continua de alta corriente

Núcleos de hierro, aluminio y bronce

Compás magnético

\section{Procedimiento}


Con los elementos de la Figura 1, construya los montajes para estudiar las leyes de la Inducción electromagnética, como se muestra en el ejemplo de la Figura 2.

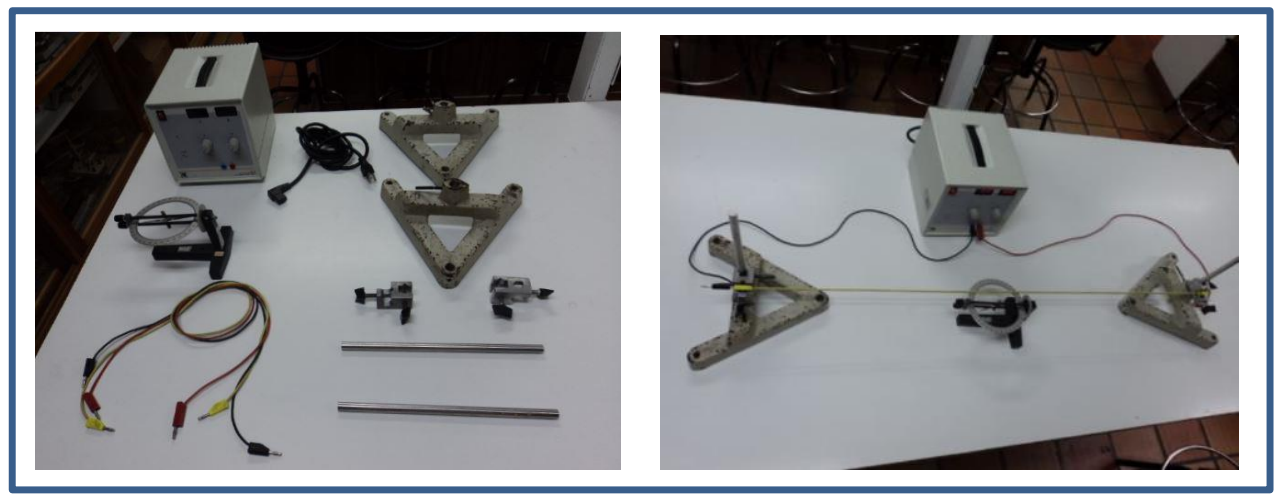

Figura 1. Materiales

Figura 2. Montaje experimental - Experimento de Oersted

A. Determinación de la polaridad del imán.

Utilizando el compás magnético colóquelo en un sitio donde no se generen campos magnéticos (no haya imanes), aprovechando el efecto del campo magnético de la tierra sobre el compás, la aguja de este va a apuntar en estas condiciones al norte geográfico (sur magnético). Entonces acerque los extremos del imán al compás y analice el comportamiento de la aguja en cada caso.

Color verde polo color rojo polo

B. Inducción de corriente (comprobación de la ley de Faraday).

Escoja un cable de los que se usa para conectar aparatos, enróllelo y forme con él las espiras, conecte los extremos del cable al galvanómetro. Con la ayuda de un compañero(a) introduzca una de las dos polaridades del imán a las espiras. Describa lo observado en el galvanómetro, si no ve cambios utilice más espiras o sea, una cables y enróllelos hasta obtener un número considerable de espiras. 
Conecte la bobina al galvanómetro (o si prefiere al multímetro), observar la deflexión de la aguja cuando se acerca el polo norte del imán a la bobina y luego cuando se retira (realizar este proceso rápidamente), indicar el sentido de la deflexión en el galvanómetro. (Dibújelos)

Realizar el planteamiento anterior introduciendo en la bobina el polo sur del imán. Indicar el sentido de la deflexión en el galvanómetro.

- Dibuje el comportamiento de las líneas de campo magnético en la bobina cuando se introduce el imán en cada polaridad.

C. Introducción de campo magnético (comprobación de la ley de Ampere).

- Utilizando de nuevo el compás magnético y ubicándolo en el lugar donde solo influya el campo magnético de la tierra, utilice la bobina secundaria conectada a la fuente y acérquela al compás. Analice el comportamiento de la aguja como se realizó en el primer paso. Identifique la dirección del campo magnético (b) que se genera por la corriente. Repita el proceso con núcleos de aluminio, cobre e hierro.

D. Generación de corriente por medio de un campo magnético inducido por otra corriente. (Unión de la ley de Ampere y ley de Faraday).

- Conecte la bobina primaria al galvanómetro y la bobina secundaria a la fuente, introduzca la bobina segundaria en la bobina primaria y observe lo que sucede en el galvanómetro.

- Ahora cambie el núcleo de aire de la bobina secundaria por un núcleo de aluminio. Realice el procedimiento anterior cambiando el núcleo de la bobina secundaria por cobre e hierro. ¿Qué diferencia se observa al introducir núcleos de diversos materiales en la bobina secundaría.

\section{Análisis}


Con sus datos experimentales:

1. ¿por qué el compás magnético muestra un comportamiento más agitado frente a uno de los polos? ¿En qué incide la distancia a la que se coloque el imán?

2. Explique la ecuación de la ley de Faraday en función de lo observado en la parte b.

3. ¿En qué influye la utilización de los diferentes núcleos en la bobina secundaria en la parte $\mathbf{d}$ de la experiencia?

4. De acuerdo a la teoría, los objetivos, el procedimiento, los datos obtenidos y calculados y sus gráficas, observe, analice y concluya sin perder de vista los aspectos físicos de este experimento.

5. Plantee tres aplicaciones relacionadas con sus estudios de ingeniería que se relacionen con el fenómeno físico estudiado en esta sesión. 


\section{Bibliografía}

Ohanian Hans c., Markert John t. Física para ingeniería y ciencias. Vol. 2 mc. Graw-Hill. Tercera edición. México, 2009.

Sears et al. Física universitaria. Vol. 2. Pearson - Addison Wesley, décimo segunda edición. México. 2009.

Serway. Jewet. Física para ciencias e ingeniería vol. 2. Thomson, sexta edición. México. 2005. 
Sesión 7- Circuitos Serie de Corriente Alterna - RLC

Temas de consulta - Circuitos Serie de Corriente Alterna - RLC (Preparación previa a la práctica)

- Circuitos de corriente alterna.

- Factor de potencia.

- Diagrama de Fasores.

\section{Competencias}

Al finalizar la práctica, el estudiante estará en capacidad de:

Verificar el comportamiento de la caída de potencial en los resistores, inductores y capacitares de un circuito de corriente alterna.

Evaluar el factor de potencia del circuito RLC implementado.

Comparar ideas y opiniones para la toma de decisiones y planes respecto a la temática estudiada junto con sus compañeros de grupo.

\section{Materiales}

- Protoboard

- Resistencia variable

- Condensador

- Inductor

- Switch

- Fuente de corriente alterna

- Osciloscopio de doble canal

- Multímetro (voltímetro y amperímetro)

- Cables

- Puntas para osciloscopio 
- Puentes

\section{Procedimiento}

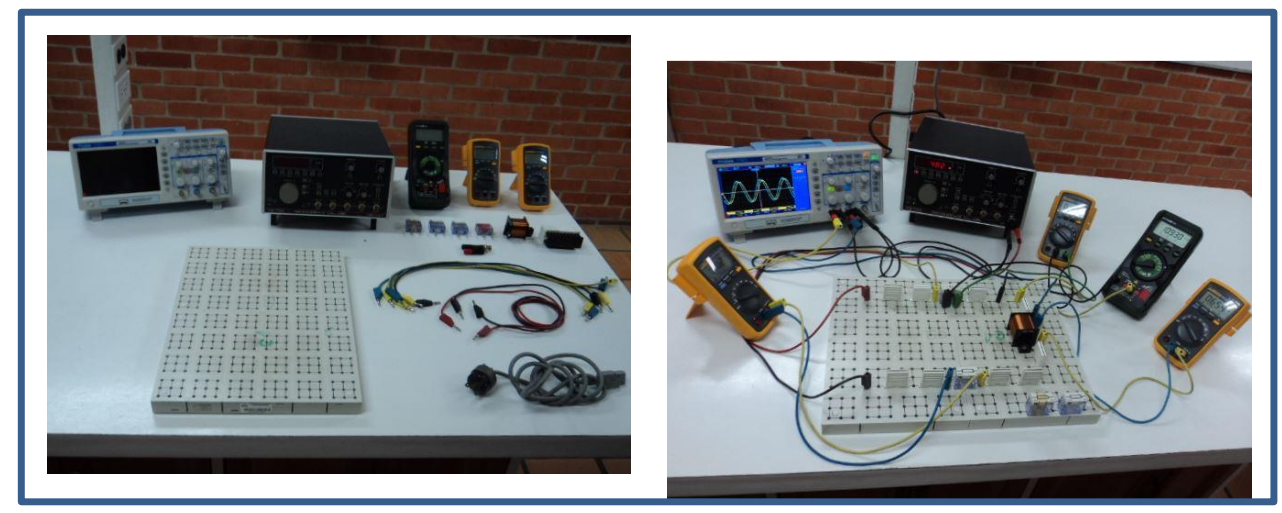

Figura 1. Materiales

Figura 2. Montaje

Con los elementos mostrados en la Figura 1 realice el montaje correspondiente al circuito RLC en Serie de corriente alterna como se muestra en la figura 2.

Nota: revise con el profesor las conexiones antes de tomar datos.

1. Implemente el circuito serie RLC de acuerdo a los valores de resistor (r), inductor (1) y capacitor

(c) proporcionados por el profesor, ver Figura 2.

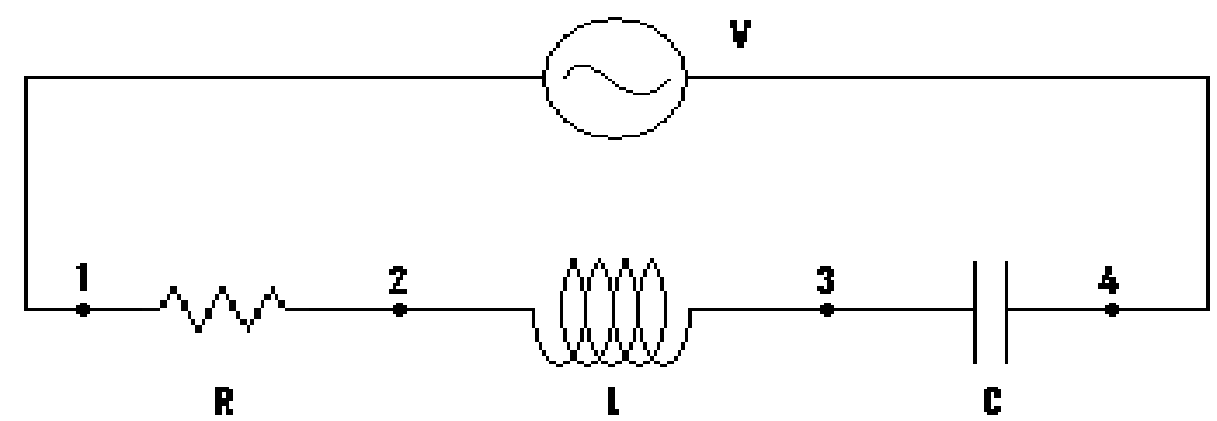

Figura 2. Esquema del circuito RLC serie de corriente alterna. 
2. Registre las amplitudes de voltaje y corriente en la siguiente tabla.

\section{Circuito RLC}

\begin{tabular}{|l|l|l|l|l|l|l|l|l|}
\hline $\mathrm{F}(\mathrm{hz})$ & $V_{r}(v)$ & $V_{l}(v)$ & $V_{c}(v)$ & $I(a)$ & $X_{l}(\Omega)$ & $X_{c}(\Omega)$ & $\phi$ & $P_{r}$ \\
\hline $\mathbf{F}<\mathbf{f}_{\mathbf{r}}$ & & & & & & & & \\
\hline $\mathbf{F r}$ & & & & & & & & \\
\hline $\mathbf{F}>\mathbf{f}_{\mathbf{r}}$ & & & & & & & & \\
\hline & & & & & & & & \\
\hline
\end{tabular}

\section{Circuito RL}

\begin{tabular}{|l|l|l|l|l|l|l|}
\hline$F(h z)$ & $V_{r}(v)$ & $V_{l}(v)$ & $I(a)$ & $X_{l}(\Omega)$ & $\phi$ & $P_{r}$ \\
\hline $\mathbf{F}<\mathbf{f}_{\mathbf{r}}$ & & & & & & \\
\hline $\mathbf{F r}$ & & & & & & \\
\hline $\mathbf{F}>\mathbf{f}_{\mathbf{r}}$ & & & & & & \\
\hline & & & & & & \\
\hline
\end{tabular}

\section{Circuito RC}

\begin{tabular}{|l|l|l|l|l|l|l|}
\hline $\boldsymbol{F}(\boldsymbol{h z})$ & $V_{r}(v)$ & $V_{c}(v)$ & $I(a)$ & $X_{c}(\Omega)$ & $\phi$ & $P_{r}$ \\
\hline $\mathbf{F}<\mathbf{f}_{\mathbf{r}}$ & & & & & & \\
\hline $\mathbf{F r}$ & & & & & & \\
\hline $\mathbf{F}>\mathbf{f}_{\mathbf{r}}$ & & & & & & \\
\hline & & & & & & \\
\hline
\end{tabular}

\section{Convenciones:}

$$
\begin{aligned}
& V=\text { voltios } \\
& A=\text { amperios } \\
& W=\text { watt } \\
& \Omega=\text { ohmios }
\end{aligned}
$$


$X_{l}=$ reactancia inductiva

$X_{c}=$ reactancia capacitiva

$\phi=$ fase

$P_{r}=$ potencia disipada en el resistor

3. Observe la diferencia de potencial en el resistor, el inductor y el capacitor. Registre la señal mediante el osciloscopio en los puntos $1-2,2-3$ y finalmente $3-4$ respectivamente.

4. Utilice ambos canales del osciloscopio, en uno de ellos registre la señal del resistor $r$, y en el otro la señal del inductor 1, observe las señales simultáneamente. Repita el procedimiento para el capacitor c.

\section{Análisis}

1. Haga un dibujo a escala de las señales observadas en el paso 3 del procedimiento. (en la hoja milimetrada).

2. Grafique sobre los mismos ejes la señal observada en el resistor y el inductor. Repita para la señal del resistor y el capacitor.

3. Realice el diagrama de Fasores con la amplitud de los voltajes en el capacitor, el inductor y el resistor de la tabla. Compare el valor del voltaje resultante con la amplitud del voltaje de la fem $(\varepsilon)$.

4. A partir de los datos registrados en la tabla y el diagrama de Fasores del paso anterior determine el factor de potencia, $\cos (\phi)$, del circuito implementado.

5. Exprese sus observaciones y conclusiones sobre los aspectos físicos de su experimento teniendo en cuenta: la teoría, el procedimiento, la tabla de datos, las gráficas y las fuentes de error.

6. Plantee tres aplicaciones relacionadas con sus estudios de ingeniería que se relacionen con el fenómeno físico estudiado en esta sesión. 


\section{Bibliografía}

Ohanian Hans c., Markert John t. Física para ingeniería y ciencias. Vol. 2 mc. Graw-Hill. Tercera edición. México, 2009.

Sears et al. Física universitaria. Vol. 2. Pearson - Addison Wesley, décimo segunda edición. México. 2009.

Serway. Jewet. Física para ciencias e ingeniería vol. 2. Thomson, sexta edición. México. 2005. 